\title{
Precision tests of CPT symmetry and quantum mechanics in the neutral kaon system
}

\author{
John Ellis, ${ }^{1}$ Jorge L. Lopez, ${ }^{2,3},{ }^{*}$ N. E. Mavromatos,,${ }^{\dagger}$ and D. V. Nanopoulos ${ }^{2,3}$ \\ ${ }^{1}$ CERN Theory Division, 1211 Geneva 23, Switzerland \\ ${ }^{2}$ Center for Theoretical Physics, Department of Physics, Texas A\&M University, College Station, Texas 77843-4242 \\ ${ }^{3}$ Astroparticle Physics Group, Houston Advanced Research Center (HARC), The Mitchell Campus, The Woodlands, Texas 77381 \\ ${ }^{4}$ Laboratoire de Physique Théorique ENSLAPP [URA 14-36 du CNRS, associée à l' E.N.S de Lyon, \\ et au LAPP (IN2P3-CNRS) d'Annecy-le-Vieux], Chemin de Bellevue, BP 110, F-74941 Annecy-le-Vieux Cedex, France
}

(Received 19 May 1995)

\begin{abstract}
We present a systematic phenomenological analysis of the tests of $C P T$ symmetry that are possible within an open quantum-mechanical description of the neutral kaon system that is motivated by arguments based on quantum gravity and string theory. We develop a perturbative expansion in terms of the three small $C P T$-violating parameters admitted in this description, and provide expressions for a complete set of $K \rightarrow 2 \pi, 3 \pi$, and $\pi \ell \nu$ decay observables to second order in these small parameters, and contrast this formalism with $C P T$ violation within conventional quantum mechanics. We also illustrate the new tests of $C P T$ symmetry and quantum mechanics that are possible in the open quantum-mechanical formalism using a regenerator. Indications are that experimental data from the CPLEAR and previous experiments could be used to establish upper bounds on the $C P T$-violating parameters that are of order $10^{-19} \mathrm{GeV}$, approaching the order of magnitude that may be attainable in quantum theories of gravity.

PACS number(s): 11.30.Er, 13.20.Eb, 14.40.Aq
\end{abstract}

\section{INTRODUCTION}

The neutral kaon system has long served as a penetrating probe of fundamental physics. It has revealed or illuminated many new areas of fundamental physics, including parity violation, $C P$ violation, flavor-changing neutral interactions, and charm. It remains the most sensitive test of fundamental symmetries, being the only place where $C P$ violation has been observed, namely, at the level of $10^{-18} \mathrm{GeV}$ in the imaginary part of the effective mass matrix for neutral kaons, and providing the most stringent microscopic check of $C P T$ symmetry within the framework of quantum mechanics, namely, $\left|\left(m_{K^{0}}-m_{\bar{K}^{0}}\right) / m_{K^{0}}\right|<9 \times 10^{-19}[1]$.

It is well known that $C P T$ symmetry is a fundamental theorem of quantum field theory, which follows from locality, unitarity, and Lorentz invariance [2]. However, the topic of $C P T$ violation has recently attracted increased attention, drawn in part by the prospect of higher-precision tests by CPLEAR [3] and at DAФNE [4], and in part by the renewed theoretical interest in quantum gravity motivated by recent developments in string theory. Some of the phenomenological discussion has been in the context of quantum mechanics [5], abandoning implicitly or explicitly the derivation of quantum mechanics from quantum field theory, in which $C P T$ is sacrosanct. Instead, we have followed the approach of Ref. [6], in which a parametrization of $C P T$-violating effects is introduced via a deviation from conventional quantum mechanics [6,7], believed to reflect the loss of quantum coherence expected in some approaches to quantum gravity

\footnotetext{
*Present address: Department of Physics, Rice University, 6100 Main Street, Houston, Texas 77005.

${ }^{\dagger}$ On leave from P.P.A.R.C. Advanced Fellowship, Department of Physics (Theoretical Physics), University of Oxford, 1 Keble Road, Oxford OX1 3NP, United Kingdom.
}

[8], notably one based on a noncritical formulation of string theory [9].

The suggestion that quantum coherence might be lost at the microscopic level was made in Ref. [8], which suggested that asymptotic scattering should be described in terms of a superscattering operator $\$$, relating initial $\left(\rho_{\text {in }}\right)$ and final $\left(\rho_{\text {out }}\right)$ density matrices, that does not factorize as a product of $S$ - and $S^{\dagger}$-matrix elements:

$$
\rho_{\text {out }}=\$ \rho_{\text {in }}: \$ \neq S S^{\dagger} .
$$

The loss of quantum coherence was thought to be a consequence of microscopic quantum-gravitational fluctuations in the space-time background. Model calculations supporting this suggestion were presented [8] as well as contested [10]. Reference [6] pointed out that if Eq. (1) is correct for asymptotic scattering, there should be a corresponding effect in the quantum Liouville equation that describes the time evolution of the dentity matrix $\rho(t)$ :

$$
\frac{\partial \rho(t)}{\partial t}=i[\rho, H]+i \delta H \rho,
$$

which is characteristic of an open quantum-mechanical system. Reference [6] parametrized the non-Hamiltonian term in the case of a simple two-state system, such as the $K^{0}-\bar{K}^{0}$ system, presented a first analysis of its phenomenological consequences, and gave experimental bounds on the nonquantum-mechanical parameters.

The question of microscopic quantum coherence has recently been addressed in the context of string theory using a variety of approaches [11]. In particular, we have analyzed this question using noncritical string theory [12], with criticality restored by nontrivial dynamics for a timelike Liouville field $[12,13]$, which we identify with the world-sheet cutoff and the target-time variable [7,9]. This approach leads to an equation of the form (2), in which probability and energy are conserved, and the possible magnitude of the ex- 
tra term $|\delta H|=O\left(E^{2} / M_{\mathrm{Pl}}\right)$, where $E$ is a typical energy scale of the system under discussion. The details of this approach are not essential for the phenomenological discussion of this paper, but it is interesting to note that the experimental sensitivity may approach this theoretical magnitude.

It has been pointed out [14] that at least the strong version of the CPT theorem must be violated in any theory described by a nonfactorizing superscattering matrix $\$(1)$, which leads to a loss of quantum coherence. This is also true of the parametrization proposed by Ref. [6], which violates $C P T$ in an intrinsically nonquantum-mechanical way. More detailed descriptions of phenomenological implications and improved experimental bounds were presented in Ref. [15]. These results were based on an analysis of $K_{L}$ and $K_{S}$ decays, and did not consider the additional constraints obtainable from an analysis of intermediate-time data. A systematic approach to the time evolution of the density matrix for the neutral kaon system was proposed in Ref. [16], and preliminary estimates of the improved experimental constraints on the nonquantum-mechanical parameters were presented. Similar results were presented later in Ref. [17], which also discussed correlation measurements possible at a $\phi$ factory such as DAФNE.

The main focus of this paper is to present detailed formulas for the time dependences of several decay asymmetries that can be measured by the CPLEAR and DAФNE experiments, using the systematic approach proposed in Ref. [16] and described in Sec. III. In particular, we discuss in Sec. IV the asymmetries known as $A_{2 \pi}, A_{3 \pi}, A_{\mathrm{T}}, A_{C P T}$, and $A_{\Delta m}$, whose definitions are reviewed in Sec. II. We show in Sec. V that experiments with a regenerator can provide useful new measurements of the nonquantum-mechanical $C P T$-violating parameters. Then, in Sec. VI we derive illustrative bounds on the nonquantum-mechanical parameters from all presently available data. Section VII contains a brief discussion of the extension of the formalism of Ref. [6] to the correlation measurements possible at $\phi$ factories such as DA $\Phi N E$. We emphasize the need to consider a general parametrization of the two-particle density matrix, that cannot be expressed simply in terms of the previously introduced single-particle density matrix parameters, and enables energy conservation to be maintained, as we have demonstrated $[7,9]$ in our noncritical string theory approach to the loss of quantum coherence. In Sec. VIII we review our conclusions, contrasting our approach with $C P T$ violation because of kaon mass or lifetime differences $\delta M, \delta \Gamma$ within conventional quantum mechanics [18] and with direct $C P T$ violation in $2 \pi$ and $\pi \ell \nu$ decay amplitudes [19], and discuss the prospects for future experimental and theoretical work. Formulas for the CPLEAR observables in the context of standard quantum-mechanical $C P T$ violation [5] are collected in Appendix A, where bounds on the corresponding parameters are also obtained. Lastly, complete formulas for the secondorder contributions to the density matrix in our quantummechanical-violating framework are collected in Appendix B.

\section{FORMALISM AND RELEVANT OBSERVABLES}

In this section we first review aspects of the modifications (2) of quantum mechanics believed to be induced by quan- tum gravity [6], as argued specifically in the context of a noncritical string analysis $[7,9]$. This provides a specific form for the modification (2) of the quantum Liouville equation for the temporal evolution of the density matrix of observable matter $[7,9]$

$$
\frac{\partial}{\partial t} \rho=i[\rho, H]+i \delta H \rho, \quad \delta H \equiv \dot{g}^{i} G_{i j}\left[g^{i}, \rho\right]
$$

where the coordinates $\left\{g^{i}\right\}$ parametrize the space of possible string models and the extra term $\delta H$ is such that the time evolution has the following basic properties.

(i) The total probability is conserved in time:

$$
\frac{\partial}{\partial t} \operatorname{Tr} \rho=0
$$

(ii) The energy $E$ is conserved on the average,

$$
\frac{\partial}{\partial t} \operatorname{Tr}(E \rho)=0
$$

as a result of the renormalizability of the world-sheet $\sigma$ model specified by the parameters $g^{i}$ which describe string propagation in a string space-time foam background.

(iii) The von Neumann entropy $S \equiv-k_{B} \operatorname{Tr} \rho \ln \rho$ increases monotonically with time,

$$
\frac{\partial}{\partial t} S \geqslant 0
$$

which vanishes only if one restricts one's attention to critical (conformal) strings, in which case there is no arrow of time $[7,9]$. However, we argue that quantum fluctuations in the background space time should be treated by including noncritical (Liouville) strings [12,13], in which case (6) becomes a strict inequality. This latter property also implies that the statistical entropy $S_{\mathrm{st}} \equiv \operatorname{Tr} \rho^{2}$ is also monotonically increasing with time, pure states evolve into mixed ones, and there is an arrow of time in this picture [7].

(iv) Correspondingly, the superscattering matrix $\$$, which is defined by its action on asymptotic density matrices,

$$
\rho_{\text {out }}=\$ \rho_{\text {in }}
$$

cannot be factorized into the usual product of the Heisenberg scattering matrix and its Hermitian conjugate,

$$
\$ \neq S S^{\dagger}, \quad S=e^{-i H t},
$$

with $H$ the Hamiltonian operator of the system. In particular, this property implies that $\$$ has no inverse, which is also expected from the property (iii).

(v) The absence of an inverse for $\$$ implies that strong $C P T$ invariance of the low-energy subsystem is lost, according to the general analysis of $[14,9]$.

It should be stressed that, although for the purposes of the present work we keep the microscopic origin of the quantum-mechanics-violating terms unspecified, it is only in the noncritical string model of Ref. [7], and the associated approach to the nature of time, that a concrete microscopic model guaranteeing the properties (i) $-(\mathrm{v})$ has so far emerged 
naturally. Within this framework, we expect that the string $\sigma$-model coordinates $g^{i}$ obey renormalization-group equations of the general form

$$
\dot{g}^{i}=\beta^{i} M_{\mathrm{Pl}}:\left|\beta^{i}\right|=O\left(\frac{E^{2}}{M_{\mathrm{Pl}}^{2}}\right)
$$

where the dot denotes differentiation with respect to the target time, measured in string $\left(M_{\mathrm{Pl}}^{-1}\right)$ units, and $E$ is a typical energy scale in the observable matter system. Since $G_{i j}$ and $g^{i}$ are themselves dimensionless numbers of order unity, we expect that in general

$$
|\delta H|=O\left(\frac{E^{2}}{M_{\mathrm{Pl}}}\right) .
$$

However, it should be emphasized that there are expected to be system-dependent numerical factors that depend on the underlying string model, and that $|\delta H|$ might further be suppressed by $\left(E / M_{\mathrm{Pl}}\right)$-dependent factors, or even vanish. Nevertheless, Eq. (10) gives us an order of magnitude to aim for in the neutral kaon system, namely, $O\left(\left(\Lambda_{\mathrm{QCD}} \text { or } m_{s}\right)^{2}\right.$ / $\left.M_{\mathrm{Pl}}\right) \sim 10^{-19} \mathrm{GeV}$. We note in passing that a phenomenologically-distinct approach to $C P T$ violation in the context of string theory is taken in [18], where it is argued that a spontaneous violation of $C P T$ may yield nonzero values of $\delta M$ and $\delta \Gamma$.

In the formalism of Ref. [6], the extra (non-Hamiltonian) term in the Liouville equation for $\rho$ can be parametrized by a $4 \times 4$ matrix $\delta H_{\alpha \beta}$, where the indices $\alpha, \beta, \ldots$ enumerate the Hermitian $\sigma$ matrices $\sigma_{0,1,2,3}$, which we represent in the $K_{1,2}$ basis. We refer the reader to the literature $[6,15]$ and Appendix A for details of this description, noting here the following forms for the neutral kaon Hamiltonian:

$$
H=\left(\begin{array}{cc}
M-\frac{i}{2} \Gamma-\operatorname{Re} M_{12}+\frac{i}{2} \operatorname{Re} \Gamma_{12} & \frac{1}{2} \delta M-\frac{i}{4} \delta \Gamma-i \operatorname{Im} M_{12}-\frac{1}{2} \operatorname{Im} \Gamma_{12} \\
\frac{1}{2} \delta M-\frac{i}{4} \delta \Gamma+i \operatorname{Im} M_{12}-\frac{1}{2} \operatorname{Im} \Gamma_{12} & M-\frac{i}{2} \Gamma+\operatorname{Re} M_{12}-\frac{i}{2} \operatorname{Re} \Gamma_{12}
\end{array}\right)
$$

in the $K_{1,2}$ basis, or

$$
H_{\alpha \beta}=\left(\begin{array}{cccc}
-\Gamma & -\frac{1}{2} \delta \Gamma & -\operatorname{Im} \Gamma_{12} & -\operatorname{Re} \Gamma_{12} \\
-\frac{1}{2} \delta \Gamma & -\Gamma & -2 \operatorname{Re} M_{12} & -2 \operatorname{Im} M_{12} \\
-\operatorname{Im} \Gamma_{12} & 2 \operatorname{Re} M_{12} & -\Gamma & -\delta M \\
-\operatorname{Re} \Gamma_{12} & -2 \operatorname{Im} M_{12} & \delta M & -\Gamma
\end{array}\right)
$$

in the $\sigma$-matrix basis. As discussed in Ref. [6], we assume that the dominant violations of quantum mechanics conserve strangeness, so that $\delta H_{1 \beta}=0$, and that $\delta H_{0 \beta}=0$ so as to conserve probability. Since $\delta H_{\alpha \beta}$ is a symmetric matrix, it follows that also $\delta H_{\alpha 0}=\delta H_{\alpha 1}=0$. Thus, we arrive at the general parametrization

$$
\delta H_{\alpha \beta}=\left(\begin{array}{cccc}
0 & 0 & 0 & 0 \\
0 & 0 & 0 & 0 \\
0 & 0 & -2 \alpha & -2 \beta \\
0 & 0 & -2 \beta & -2 \gamma
\end{array}\right)
$$

where, as a result of the positivity of the Hermitian density matrix $\rho[6]$,

$$
\alpha, \gamma>0, \quad \alpha \gamma>\beta^{2}
$$

We recall [15] that the $C P T$ transformation can be expressed as a linear combination of $\sigma_{2,3}$ in the $K_{1,2}$ basis: $C P T=\sigma_{3} \cos \theta+\sigma_{2} \sin \theta$, for some choice of phase $\theta$. It is apparent that none of the nonzero terms $\propto \alpha, \beta, \gamma$ in $\delta H_{\alpha \beta}$ (13) commutes with the $C P T$ transformation. In other words, each of the three parameters $\alpha, \beta, \gamma$ violates $C P T$, leading to a richer phenomenology than that in conventional quantum mechanics. This is because the symmetric $\delta H$ matrix has three parameters in its bottom right-hand $2 \times 2$ submatrix, whereas the $h$ matrix appearing in the time evolution within quantum mechanics [5] has only one complex $C P T$-violating parameter $\delta$,

$$
\delta=-\frac{1}{2} \frac{\frac{1}{2} \delta \Gamma+i \delta M}{\frac{1}{2}|\Delta \Gamma|+i \Delta m}
$$

where $\delta M$ and $\delta \Gamma$ violate $C P T$, but do not induce any mixing in the time evolution of pure state vectors [15]. The parameters $\Delta m=M_{L}-M_{S}$ and $|\Delta \Gamma|=\Gamma_{S}-\Gamma_{L}$ are the usual differences between mass and decay widths, respectively, of $K_{L}$ and $K_{S}$ states. A brief review of the quantum-mechanical formalism is given in Appendix A. For more details, we refer the reader to the literature $[18,19]$. The above results imply that the experimental constraints [1] on $C P T$ violation have to be rethought if one allows a modification of quantum mechanics, as discussed here. As we shall discuss later on, there are essential differences between quantum-mechanical $C P T$ violation and the nonquantum-mechanical $C P T$ violation induced by the effective parameters $\alpha, \beta, \gamma[6]$.

Useful observables are associated with the decays of neutral kaons to $2 \pi$ or $3 \pi$ final states, or semileptonic decays to $\pi l \nu$. In the density-matrix formalism introduced above, their values are given by expressions of the form [6] 


$$
\left\langle O_{i}\right\rangle=\operatorname{Tr}\left[O_{i} \rho\right],
$$

where the observables $O_{i}$ are represented by $2 \times 2$ Hermitian matrices. For future use, we give their expressions in the $K_{1,2}$ basis:

$$
\begin{gathered}
O_{2 \pi}=\left(\begin{array}{ll}
0 & 0 \\
0 & 1
\end{array}\right), \quad O_{3 \pi^{\circ}} \propto\left(\begin{array}{ll}
1 & 0 \\
0 & 0
\end{array}\right), \\
O_{\pi^{-} l^{+} \nu}=\left(\begin{array}{ll}
1 & 1 \\
1 & 1
\end{array}\right), \quad O_{\pi^{+} l^{--} \nu}=\left(\begin{array}{cc}
1 & -1 \\
-1 & 1
\end{array}\right),
\end{gathered}
$$

which constitute a complete Hermitian set. As we discuss in more detail later, it is possible to measure the interference between $K_{1,2}$ decays into $\pi^{+} \pi^{-} \pi^{0}$ final states with different
$C P$ properties, by restricting one's attention to part of the phase space $\Omega$, e.g., final states with $m\left(\pi^{+} \pi^{0}\right)>m\left(\pi^{-} \pi^{0}\right)$. In order to separate this interference from that because of $K_{S, L}$ decays into final states with identical $C P$ properties, because of $C P$ violation in the $K_{1,2}$ mass matrix or in decay amplitudes, we consider [20] the difference between final states with $m\left(\pi^{+} \pi^{0}\right)>m\left(\pi^{-} \pi^{0}\right)$ and $m\left(\pi^{+} \pi^{0}\right)<m\left(\pi^{-} \pi^{0}\right)$. This observable is represented by the matrix

$$
O_{3 \pi}^{\mathrm{int}}=\left(\begin{array}{cc}
0 & \mathscr{K} \\
\mathscr{K}^{*} & 0
\end{array}\right)
$$

where

$$
\mathscr{K} \equiv \frac{\left[\int_{m\left(\pi^{+} \pi^{0}\right)>m\left(\pi^{-} \pi^{0}\right)} d \Omega-\int_{m\left(\pi^{+} \pi^{0}\right)<m\left(\pi^{-} \pi^{0}\right)} d \Omega\right] A_{2}\left(I_{3 \pi}=2\right) A_{1}\left(I_{3 \pi}=1\right)}{\int d \Omega\left|A_{1}\left(I_{3 \pi}=1\right)\right|^{2}},
$$

where $\mathscr{K}$ is expected to be essentially real, so that the $O_{3 \pi}^{\text {int }}$ observable provides essentially the same information as $O_{\pi^{-} l^{+} \nu}-O_{\pi^{+} l^{-} \bar{\nu}}$.

In this formalism, pure $K^{0}$ or $\bar{K}^{0}$ states, such as the ones used as initial conditions in the CPLEAR experiment [3], are described by the density matrices

$$
\rho_{K^{0}}=\frac{1}{2}\left(\begin{array}{ll}
1 & 1 \\
1 & 1
\end{array}\right), \quad \rho_{\bar{K}^{0}}=\frac{1}{2}\left(\begin{array}{cc}
1 & -1 \\
-1 & 1
\end{array}\right) .
$$

We note the similarity of the above density matrices (21) to the semileptonic decay observables in (18), which is because of the strange quark $(s)$ content of the kaon $K^{0}$ $\ni \bar{s} \rightarrow \bar{u} l^{+} \nu, \bar{K}^{0} \ni s \rightarrow u l^{-} \bar{\nu}$, and our assumption of the validity of the $\Delta S=\Delta Q$ rule.

In this paper we shall apply the above formalism to compute the time evolution of certain experimentally observed quantities that are of relevance to the CPLEAR experiment [3]. These are asymmetries associated with decays of an initial $K^{0}$ beam as compared to corresponding decays of an initial $\bar{K}^{0}$ beam:

$$
A(t)=\frac{R\left(\bar{K}_{t=0}^{0} \rightarrow \bar{f}\right)-R\left(K_{t=0}^{0} \rightarrow f\right)}{R\left(\bar{K}_{t=0}^{0} \rightarrow \bar{f}\right)+R\left(K_{t=0}^{0} \rightarrow f\right)},
$$

where $R\left(K^{0} \rightarrow f\right) \equiv \operatorname{Tr}\left[O_{f} \rho(t)\right]$ denotes the decay rate into the final state $f$, given that one starts from a pure $K^{0}$ at $t=0$, whose density matrix is given in (21), and $R\left(\bar{K}^{0} \rightarrow \bar{f}\right) \equiv \operatorname{Tr}\left[O_{f} \bar{\rho}(t)\right]$ denotes the decay rate into the conjugate state $\bar{f}$, given that one starts from a pure $\bar{K}^{0}$ at $t=0$.

Let us illustrate the above formalism by two examples. We may compute the asymmetry for the case where there are identical final states $f=\bar{f}=2 \pi$, in which case the observable is given in (17). We obtain

$$
A_{2 \pi}=\frac{\operatorname{Tr}\left[O_{2 \pi} \bar{\rho}(t)\right]-\operatorname{Tr}\left[O_{2 \pi} \rho(t)\right]}{\operatorname{Tr}\left[O_{2 \pi} \bar{\rho}(t)\right]+\operatorname{Tr}\left[O_{2 \pi} \rho(t)\right]}=\frac{\operatorname{Tr}\left[O_{2 \pi} \Delta \rho(t)\right]}{\operatorname{Tr}\left[O_{2 \pi} \Sigma \rho(t)\right]},
$$

where we have defined: $\Delta \rho(t) \equiv \bar{\rho}(t)-\rho(t)$ and $\Sigma \rho(t) \equiv \bar{\rho}(t)+\rho(t)$. We note that in the above formalism we make no distinction between neutral and charged two-pion final states. This is because we neglect, for simplicity, the effects of $\epsilon^{\prime}$. Since $\left|\epsilon^{\prime} / \epsilon\right| \lesssim 10^{-3}$, this implies that our analysis of the new quantum-mechanics-violating parameters must be refined if magnitudes $\lesssim \epsilon^{\prime}|\Delta \Gamma| \simeq 10^{-6}|\Delta \Gamma|$ are to be studied.

In a similar spirit to the identical final state case, one can compute the asymmetry $A_{\mathrm{T}}$ for the semileptonic decay case, where $f=\pi^{+} l^{-} \bar{\nu} \neq \bar{f}=\pi^{-} l^{+} \nu$. The formula for this observable is

$$
A_{\mathrm{T}}(t)=\frac{\operatorname{Tr}\left[O_{\pi^{-} l^{+} \nu} \bar{\rho}(t)\right]-\operatorname{Tr}\left[O_{\pi^{+} l^{-} \bar{\nu}} \rho(t)\right]}{\operatorname{Tr}\left[O_{\pi^{-} l^{+} \nu} \bar{\rho}(t)\right]+\operatorname{Tr}\left[O_{\pi^{+} l^{-} \bar{\nu}} \rho(t)\right]} .
$$

Other observables are discussed in Sec. IV.

To determine the temporal evolution of the above observables, which is crucial for experimental fits, it is necessary to know the equations of motion for the components of $\rho$ in the $K_{1,2}$ basis. These are $[6,15]^{1}$

$$
\begin{gathered}
\dot{\rho}_{11}=-\Gamma_{L} \rho_{11}+\gamma \rho_{22}-2 \operatorname{Re}\left[\left(\operatorname{Im} M_{12}-i \beta\right) \rho_{12}\right], \\
\dot{\rho}_{12}=-(\Gamma+i \Delta m) \rho_{12}-2 i \alpha \operatorname{Im} \rho_{12} \\
+\left(\operatorname{Im} M_{12}-i \beta\right)\left(\rho_{11}-\rho_{22}\right), \\
\dot{\rho}_{22}=-\Gamma_{S} \rho_{22}+\gamma \rho_{11}+2 \operatorname{Re}\left[\left(\operatorname{Im} M_{12}-i \beta\right) \rho_{12}\right],
\end{gathered}
$$

\footnotetext{
${ }^{1}$ Since we neglect $\epsilon^{\prime}$ effects and assume the validity of the $\Delta S$ $=\Delta Q$ rule, in what follows we also consistently neglect $\operatorname{Im} \Gamma_{12}[4]$.
} 
where, for instance, $\rho$ may represent $\Delta \rho$ or $\Sigma \rho$, defined by the initial conditions

$$
\Delta \rho(0)=\left(\begin{array}{cc}
0 & -1 \\
-1 & 0
\end{array}\right), \quad \Sigma \rho(0)=\left(\begin{array}{ll}
1 & 0 \\
0 & 1
\end{array}\right) .
$$

In these equations, $\Gamma_{L}=\left(5.17 \times 10^{-8} \mathrm{~s}\right)^{-1}$ and $\Gamma_{S}=\left(0.8922 \times 10^{-10} \mathrm{~s}\right)^{-1}$ are the inverse $K_{L}$ and $K_{S}$ lifetimes, $\quad \Gamma \equiv\left(\Gamma_{S}+\Gamma_{L}\right) / 2, \quad|\Delta \Gamma| \equiv \Gamma_{S}-\Gamma_{L}=(7.364 \pm 0.016)$ $\times 10^{-15} \mathrm{GeV}$, and $\Delta m=0.5351 \times 10^{10} \mathrm{~s}^{-1}=3.522 \times 10^{-15}$ $\mathrm{GeV}$ is the $K_{L}-K_{S}$ mass difference. Also, the $C P$ impurity parameter $\epsilon$ is given by

$$
\epsilon=\frac{\operatorname{Im} M_{12}}{\frac{1}{2}|\Delta \Gamma|+i \Delta m},
$$

which leads to the relations

$$
\operatorname{Im} M_{12}=\frac{1}{2} \frac{|\Delta \Gamma||\epsilon|}{\cos \phi}, \quad \epsilon=|\epsilon| e^{-i \phi}: \tan \phi=\frac{\Delta m}{\frac{1}{2}|\Delta \Gamma|},
$$

with $|\epsilon| \approx 2.2 \times 10^{-3}$ and $\phi \approx 45^{\circ}$ the "superweak" phase.

These equations are to be compared with the corresponding quantum-mechanical equations of Refs. $[5,15]$ which are reviewed in Appendix A. The parameters $\delta M$ and $\beta$ play similar roles, although they appear with different relative signs in different places, because of the symmetry of $\delta H$ as opposed to the antisymmetry of the quantum-mechanical evolution matrix $H$. These differences are important for the asymptotic limits of the density matrix, and its impurity. In our approach, one can readily show that, at large $t, \rho$ decays exponentially to [15]

$$
\rho_{L} \approx\left(\begin{array}{cc}
1 & (|\epsilon|+i 2 \hat{\beta} \cos \phi) e^{i \phi} \\
(|\epsilon|-i 2 \hat{\beta} \cos \phi) e^{-i \phi} & |\epsilon|^{2}+\hat{\gamma}-4 \hat{\beta}^{2} \cos ^{2} \phi-4 \hat{\beta}|\epsilon| \sin \phi
\end{array}\right),
$$

where we have defined the following scaled variables

$$
\hat{\alpha}=\alpha /|\Delta \Gamma|, \quad \hat{\beta}=\beta /|\Delta \Gamma|, \quad \hat{\gamma}=\gamma /|\Delta \Gamma| .
$$

Conversely, if we look in the short-time limit for a solution of Eqs. (25)-(27) $\rho_{11} \ll \rho_{12} \ll \rho_{22}$, we find [15]

$$
\rho_{S} \approx\left(\begin{array}{cc}
|\epsilon|^{2}+\hat{\gamma}-4 \hat{\beta}^{2} \cos ^{2} \phi+4 \hat{\beta}|\epsilon| \sin \phi & (|\epsilon|+i 2 \hat{\beta} \cos \phi) e^{-i \phi} \\
(|\epsilon|-i 2 \hat{\beta} \cos \phi) e^{i \phi} & 1
\end{array}\right) .
$$

These results are to be contrasted with those obtained within conventional quantum mechanics,

$$
\rho_{L} \approx\left(\begin{array}{cc}
1 & \epsilon^{*} \\
\epsilon & |\epsilon|^{2}
\end{array}\right), \quad \rho_{S} \approx\left(\begin{array}{cc}
|\epsilon|^{2} & \epsilon \\
\epsilon^{*} & 1
\end{array}\right)
$$

which, as can be seen from their vanishing determinant, ${ }^{2}$ correspond to pure $K_{L}$ and $K_{S}$ states, respectively. In contrast, $\rho_{L}, \rho_{S}$ in Eqs. (31) and (33) describe mixed states. As mentioned in the Introduction, the maximum possible order of magnitude for $|\alpha|,|\beta|$, or $|\gamma|$ that we could expect theoretically is $O\left(E^{2} / M_{\mathrm{Pl}}\right)=O\left(\left(\Lambda_{\mathrm{QCD}} \text { orm }_{s}\right)^{2} / M_{\mathrm{Pl}}\right) \sim 10^{-19}$ $\mathrm{GeV}$ in the neutral kaon system.

To make a consistent phenomenological study of the various quantities discussed above, it is essential to solve the coupled system of equations (25)-(27) for intermediate times. This requires approximations in order to get analytic results [16], as we discuss in the next section.

\footnotetext{
${ }^{2}$ A pure state will remain pure as long as $\operatorname{Tr} \rho^{2}=(\operatorname{Tr} \rho)^{2}$ [6]. In the case of $2 \times 2$ matrices $\operatorname{Tr} \rho^{2}=(\operatorname{Tr} \rho)^{2}-2 \operatorname{det} \rho$, and therefore the purity condition is equivalently expressed as $\operatorname{det} \rho=0$.
}

\section{PERTURBATION THEORY}

The coupled set of differential equations (25)-(27) can be solved numerically to any desired degree of accuracy. However, it is instructive and adequate for our purposes to solve these equations in perturbation theory in $\hat{\alpha}, \hat{\beta}, \hat{\gamma}$, and $|\epsilon|$, so as to obtain convenient analytical approximations [16]. Writing

$$
\rho_{i j}(t)=\rho_{i j}^{(0)}(t)+\rho_{i j}^{(1)}(t)+\rho_{i j}^{(2)}(t)+\cdots,
$$

where $\rho_{i j}^{(n)}(t)$ is proportional to $\hat{\alpha}^{p_{\alpha}} \hat{\beta}^{p_{\beta}} \hat{\gamma}^{p_{\gamma}}|\epsilon|^{p_{\epsilon}}$, with $p_{\alpha}+p_{\beta}+p_{\gamma}+p_{\epsilon}=n$, we obtain a set of differential equations at each order in perturbation theory. To zeroth order we get

$$
\begin{gathered}
\rho_{11}^{(0)}(t)=\rho_{11}(0) e^{-\Gamma_{L^{t}},} \\
\rho_{22}^{(0)}(t)=\rho_{22}(0) e^{-\Gamma_{S} t}, \\
\rho_{12}^{(0)}(t)=\rho_{12}(0) e^{-(\Gamma+i \Delta m t),}
\end{gathered}
$$

where, in the interest of generality, we have left the initial conditions unspecified. At higher orders, the differential equations are of the form 


$$
\dot{\rho}_{i j}^{(n)}(t)=-A \rho_{i j}^{(n)}(t)+\sum_{i^{\prime} j^{\prime}}{ }^{\prime} \rho_{i^{\prime} j^{\prime}}^{(n-1)}(t),
$$

where $\Sigma^{\prime}$ excludes the $\rho_{i j}$ term. Multiplying by the integrating factor $e^{A t}$, one obtains

$$
\frac{d}{d t}\left[e^{A t} \rho_{i j}^{(n)}(t)\right]=e^{A t} \sum_{i^{\prime} j^{\prime}}{ }^{\prime} \rho_{i^{\prime} j^{\prime}}^{(n-1)}(t),
$$

which can be integrated in terms of the known functions at the $(n-1)$ th order, and the initial condition $\rho_{i j}^{(n)}(0)=0$, for $n \geqslant 1$ : i.e.,

$$
\rho_{i j}^{(n)}(t)=e^{-A t} \int_{0}^{t} d t^{\prime} e^{A t^{\prime}} \sum_{i^{\prime} j^{\prime}}^{\prime} \rho_{i^{\prime} j^{\prime}}^{(n-1)}\left(t^{\prime}\right) .
$$

Following this straightforward (but tedious) procedure, we obtain the set of first-order expressions

$$
\begin{aligned}
& \rho_{11}^{(1)}(t)=\rho_{22}(0) \hat{\gamma}\left[e^{-\Gamma_{L^{t}}}-e^{-\Gamma_{S} t}\right] \\
& +\frac{2|\epsilon|}{\cos \delta \phi}\left|\rho_{12}(0)\right|\left[e^{-\Gamma t} \cos \left(\Delta m t+\phi-\delta \phi-\phi_{12}\right)\right. \\
& \left.-e^{-\Gamma_{L} t} \cos \left(\phi-\delta \phi-\phi_{12}\right)\right], \\
& \rho_{22}^{(1)}(t)=\rho_{11}(0) \hat{\gamma}\left[e^{-\Gamma_{L} t}-e^{-\Gamma_{S} t}\right] \\
& +\frac{2|\epsilon|}{\cos \delta \phi}\left|\rho_{12}(0)\right|\left[e^{-\Gamma t} \cos \left(\Delta m t-\phi-\delta \phi-\phi_{12}\right)\right. \\
& \left.-e^{-\Gamma_{S} t} \cos \left(\phi+\delta \phi+\phi_{12}\right)\right], \\
& \rho_{12}^{(1)}(t)=\frac{2 \hat{\alpha}}{\tan \phi}\left|\rho_{12}(0)\right| e^{-\Gamma t}\left[e^{-i \phi_{12} \sin (\Delta m t)}\right. \\
& \left.-(\Delta m t) e^{-i \Delta m t+i \phi_{12}}\right]+\frac{|\epsilon|}{\cos \delta \phi}\left\{\rho_{11}(0) e^{i(\delta \phi-\phi)}\right. \\
& \times\left[e^{-\Gamma_{L} t}-e^{-(\Gamma+i \Delta m) t}\right]+\rho_{22}(0) e^{i(\delta \phi+\phi)} \\
& \left.\times\left[e^{-\Gamma_{S} t}-e^{-(\Gamma+i \Delta m) t}\right]\right\} .
\end{aligned}
$$

In these expressions, $\phi_{12}=\operatorname{Arg}\left[\rho_{12}(0)\right]$, and we have defined

$$
\tan \delta \phi=-\frac{2 \hat{\beta} \cos \phi}{|\epsilon|}
$$

Note that generically all three parameters $(\hat{\alpha}, \hat{\beta}, \hat{\gamma})$ appear to first order. However, in the specific observables to be discussed below, this is not necessarily the case because of the particular initial conditions that may be involved. Thus, these general expressions may be useful in the design of experiments that seek to maximize the sensitivity to the $C P T$-violating parameters. To obtain the expressions for
$\Delta \rho$ and $\Sigma \rho$, one simply needs to insert the appropriate set of initial conditions [Eq. (28)]. Through first order we obtain the ready-to-use expressions

$$
\begin{aligned}
& \Delta \rho_{11}^{(0+1)}(t)=\frac{2|\epsilon|}{\cos \delta \phi}\left[-e^{-\Gamma t} \cos (\Delta m t+\phi-\delta \phi)\right. \\
& \left.+e^{-\Gamma_{L} t} \cos (\phi-\delta \phi)\right], \\
& \Delta \rho_{22}^{(0+1)}(t)=\frac{2|\epsilon|}{\cos \delta \phi}\left[-e^{-\Gamma t} \cos (\Delta m t-\phi-\delta \phi)\right. \\
& \left.+e^{-\Gamma_{S} t} \cos (\phi+\delta \phi)\right], \\
& \Delta \rho_{12}^{(0+1)}(t)=-e^{-(\Gamma+i \Delta m) t}-\frac{2 \hat{\alpha}}{\tan \phi} e^{-\Gamma t}[\sin (\Delta m t) \\
& \left.-(\Delta m t) e^{-i \Delta m t}\right], \\
& \Sigma \rho_{11}^{(0+1)}(t)=e^{-\Gamma_{L} t}+\hat{\gamma}\left[e^{-\Gamma_{L} t}-e^{-\Gamma_{S} t}\right], \\
& \Sigma \rho_{22}^{(0+1)}(t)=e^{-\Gamma_{S} t}+\hat{\gamma}\left[e^{-\Gamma_{L} t}-e^{-\Gamma_{S} t}\right], \\
& \Sigma \rho_{12}^{(0+1)}(t)=\frac{|\epsilon|}{\cos \delta \phi}\left\{e^{i(\delta \phi-\phi)}\left[e^{-\Gamma_{L} t}-e^{-(\Gamma+i \Delta m) t}\right]\right. \\
& \left.+e^{i(\delta \phi+\phi)}\left[e^{-\Gamma_{S} t}-e^{-(\Gamma+i \Delta m) t}\right]\right\} .
\end{aligned}
$$

For most purposes, first-order approximations suffice. However, in the case of the $A_{2 \pi}$ and $R_{2 \pi}$ observables, some second-order terms in the expression for $\rho_{22}$ are required. For example, $\Delta \rho_{22}^{(2)}$ introduces the first $\alpha$ dependence in the numerator of $A_{2 \pi}$, whereas $\Sigma \rho_{22}^{(2)}$ cuts off the otherwise exponential growth with time of the numerator. The complete second-order expressions for $\rho_{11,22,12}$ are collected in Appen$\operatorname{dix} B$.

\section{ANALYTICAL RESULTS}

We now proceed to give explicit expressions for the temporal evolution of the asymmetries $A_{2 \pi}, A_{3 \pi}, A_{\mathrm{T}}$, $A_{C P T}$, and $A_{\Delta m}$ that are possible objects of experimental study, in particular, by the CPLEAR Collaboration [3].

\section{A. $A_{2 \pi}$}

Following the discussion in Sec. II, one obtains, for this asymmetry,

$$
A_{2 \pi}(t)=\frac{\Delta \rho_{22}(t)}{\sum \rho_{22}(t)}
$$

with $\Delta \rho_{22}$ and $\Sigma \rho_{22}$ given through first order in Eqs. (47) and (50); second-order contributions can be obtained from Eq. (B16). The result for the asymmetry, to second order in the small parameters, can be written most concisely as

$$
A_{2 \pi}(t)=\frac{\left[2|\epsilon| \frac{\cos (\phi+\delta \phi)}{\cos \delta \phi}+\Delta X_{1}\right]+e^{\left(\Gamma_{S}-\Gamma_{L}\right) t} \Delta X_{2}-e^{1 / 2\left(\Gamma_{S}-\Gamma_{L}\right) t}\left[\frac{2|\epsilon|}{\cos \delta \phi} \cos (\Delta m t-\phi-\delta \phi)+\Delta X_{3}\right]}{\left[1-\hat{\gamma}+\Sigma X_{1}\right]+e^{\left(\Gamma_{S}-\Gamma_{L}\right) t}\left[\hat{\gamma}+\Sigma X_{2}\right]-e^{1 / 2\left(\Gamma_{S}-\Gamma_{L}\right) t} \Sigma X_{3}},
$$



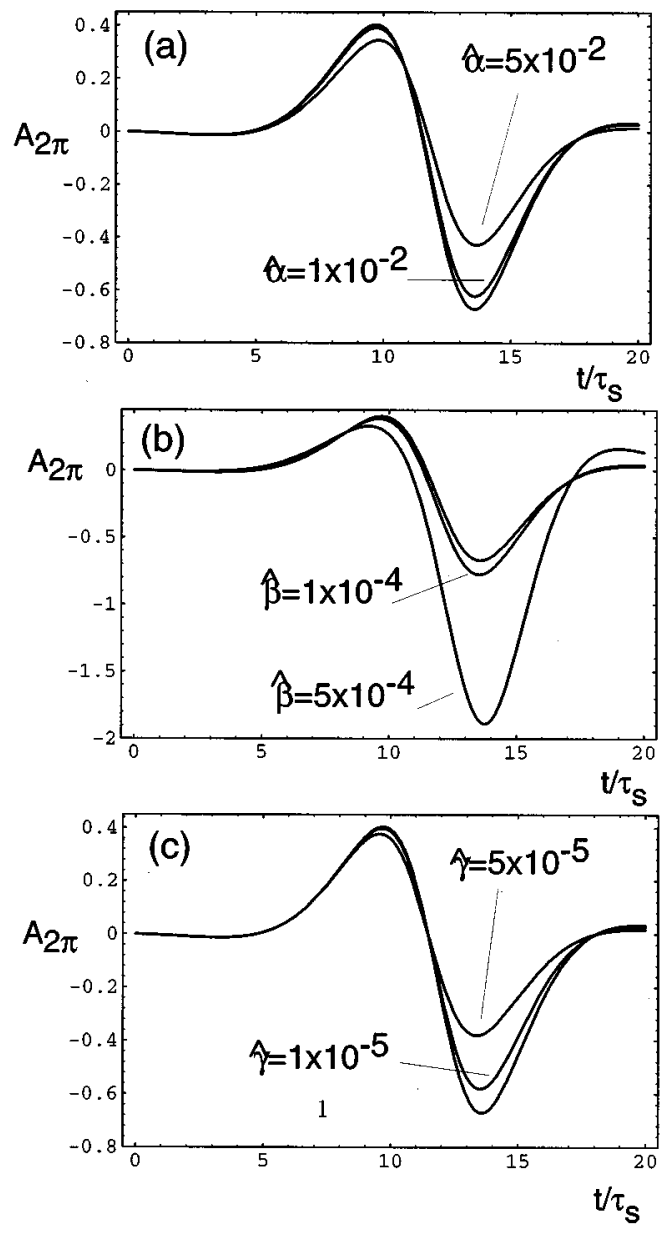

FIG. 1. The time-dependent asymmetry $A_{2 \pi}$ for various choices of the $C P T$-violating parameters: (a) dependence on $\hat{\alpha}$, (b) dependence on $\hat{\beta}$, (c) dependence on $\hat{\gamma}$. The unspecified parameters are set to zero. The curve with no label corresponds to the standard case $(\hat{\alpha}=\hat{\beta}=\hat{\gamma}=0)$.

where the second-order coefficients $\Delta X_{1,2,3}$ and $\Sigma X_{1,2,3}$ are given by

$$
\Delta X_{1}=2|\epsilon| \hat{\gamma} \frac{\cos (\phi+\delta \phi)}{\cos \delta \phi}-8 \hat{\alpha}|\epsilon| \sin \phi \cos \phi \frac{\sin (\phi+\delta \phi)}{\cos \delta \phi}
$$

$$
\Delta X_{2}=2|\epsilon| \hat{\gamma} \frac{\cos (\phi-\delta \phi)}{\cos \delta \phi}
$$

$$
\begin{aligned}
\Delta X_{3}= & 4|\epsilon| \hat{\gamma} \frac{\cos \phi}{\cos \delta \phi} \cos (\Delta m t-\delta \phi)+\frac{4|\epsilon| \hat{\alpha}}{\tan \phi} \sin (\Delta m t-\phi) \\
& -4|\epsilon| \hat{\alpha} \frac{\cos \phi}{\cos \delta \phi}\left[\frac{t|\Delta \Gamma|}{2 \cos \phi} \cos (\Delta m t-\phi-\delta \phi)\right. \\
& -\cos (\Delta m t-2 \phi-\delta \phi)]
\end{aligned}
$$
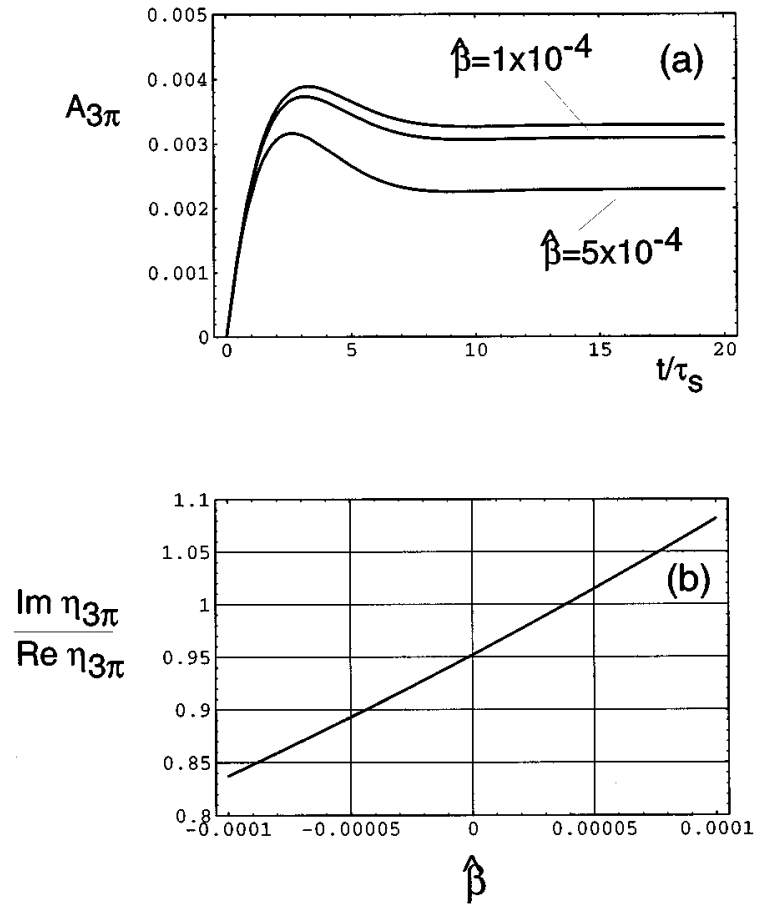

FIG. 2. The time-dependent asymmetry $A_{3 \pi}$ for representative choices of $\hat{\beta}\left(A_{3 \pi}\right.$ depends very weakly on $\left.\hat{\alpha}, \hat{\gamma}\right)$. The top curve corresponds to the standard case. Also shown is the ratio $\operatorname{Im} \eta_{3 \pi} / \operatorname{Re} \eta_{3 \pi}=\tan (\phi-\delta \phi)$ as a function of $\hat{\beta}$.

$$
\begin{gathered}
\Sigma X_{1}=-\hat{\gamma}^{2}+\frac{2|\epsilon|^{2}}{\cos ^{2} \delta \phi}[\cos (2 \delta \phi)+\cos (2 \phi+2 \delta \phi) \\
\left.-\frac{\cos (\phi-2 \delta \phi)}{2 \cos \phi}\right] \\
+t|\Delta \Gamma|\left[-\hat{\gamma}^{2}+|\epsilon|^{2} \frac{\cos (\phi+2 \delta \phi)}{\cos \phi \cos ^{2} \delta \phi}\right] \\
\Sigma X_{2}=\hat{\gamma}^{2}+|\epsilon|^{2} \frac{\cos (\phi-2 \delta \phi)}{\cos \phi \cos ^{2} \delta \phi}
\end{gathered}
$$

$$
\Sigma X_{3}=\frac{2|\epsilon|^{2}}{\cos ^{2} \delta \phi}[\cos (\Delta m t-2 \delta \phi)+\cos (\Delta m t-2 \phi-2 \delta \phi)] .
$$

This form is useful when $\hat{\beta} \ll|\epsilon|$, since then $\delta \phi \approx 0$. In the usual case (i.e., $\hat{\alpha}=\hat{\beta}=\hat{\gamma}=0$ ), we obtain

$$
A_{2 \pi}(t)=\frac{2|\epsilon| \cos \phi-2|\epsilon| e^{1 / 2\left(\Gamma_{S}-\Gamma_{L}\right) t} \cos (\Delta m t-\phi)}{\left[1+\sum X_{1}^{u}\right]+e^{\left(\Gamma_{S}-\Gamma_{L}\right) t} \sum X_{2}^{u}-e^{1 / 2\left(\Gamma_{S}-\Gamma_{L}\right) t} \sum X_{3}^{u}},
$$

with

$$
\begin{gathered}
\Sigma X_{1}^{u}=|\epsilon|^{2}[1+2 \cos (2 \phi)+t|\Delta \Gamma|], \\
\Sigma X_{2}^{u}=|\epsilon|^{2}, \\
\Sigma X_{3}^{u}=4|\epsilon|^{2} \cos \phi \cos (\Delta m t-\phi) .
\end{gathered}
$$


Comparing the two cases, we note the following.

(1) The second line in Eq. (53) shows that (to first order) $\delta \phi \neq 0$ changes the size of the interference pattern and shifts it.

(2) The denominator in Eq. (53) shows that necessarily $\hat{\gamma} \leqslant \Sigma X_{2} \sim|\epsilon|^{2}$, or else the interference pattern would be damped too soon. In fact, because of this upper limit, one can in practice neglect all terms proportional to $\hat{\gamma}$ that appear formally at second order, since they are in practice third order.

(3) The effect of $\hat{\alpha}$ is felt only at second order, through $\Delta X_{1}$ and $\Delta X_{3}$, although it is of some relevance only in the interference pattern $\left(\Delta X_{3}\right)$.

Some of the terms in Eq. (53) can be written in a less concise way which shows the effect of $\hat{\beta}$ more explicitly, instead of it being buried inside $\delta \phi$. To first order, although keeping the important second-order terms in $\Sigma X_{2}$, we can write

$$
\begin{aligned}
A_{2 \pi}(t) \approx & \left\{2|\epsilon| \cos \phi+4 \hat{\beta} \sin \phi \cos \phi-2 \sqrt{|\epsilon|^{2}+4 \hat{\beta}^{2} \cos ^{2} \phi}\right. \\
& \left.\times e^{1 / 2\left(\Gamma_{S}-\Gamma_{L}\right) t} \cos (\Delta m t-\phi-\delta \phi)\right\} \\
& \times\left\{1+e^{\left(\Gamma_{S}-\Gamma_{L}\right) t}\left[\hat{\gamma}+|\epsilon|^{2}-4 \hat{\beta}^{2} \cos ^{2} \phi\right.\right. \\
& -4 \hat{\beta}|\epsilon| \sin \phi]\}
\end{aligned}
$$

In this form one can readily see whether $C P$ violation can in fact vanish, with its effects mimicked by nonquantummechanical $C P T$ violation. Setting $|\epsilon|=0$, one needs to reproduce the interference pattern, and also the denominator. To reproduce the overall coefficient of the interference pat- tern, requires $2 \hat{\beta} \cos \phi \rightarrow \pm|\epsilon|$. The denominator then becomes $\hat{\gamma}-4 \hat{\beta}^{2} \cos ^{2} \phi \rightarrow \hat{\gamma}-|\epsilon|^{2}$ and we also require $\hat{\gamma} \rightarrow 2|\epsilon|^{2}$. The fatal problem is that $\delta \phi \rightarrow-\operatorname{sgn}(\hat{\beta}) \frac{\pi}{2}$ and the interference pattern is shifted significantly. This means that the effects seen in the neutral kaon system, and conventionally interpreted as $C P$ violation, indeed cannot because of the $C P T$ violation $[16,17]$.

Figure 1 shows the effects on $A_{2 \pi}(t)$ of varying (a) $\hat{\alpha}$, (b) $\hat{\beta}$, and (c) $\hat{\gamma}$. We see that the intermediate-time region 5 $\lesssim t / \tau_{s} \lesssim 20$ is particularly sensitive to nonzero values of these parameters. The sensitivity to $\hat{\alpha}$ in Fig. 1(a) is considerably less than that to $\hat{\beta}$ in Fig. 1(b) and to $\hat{\gamma}$ in Fig. 1(c), which is reflected in the magnitudes of the indicative numerical bounds reported in Sec. VI.

$$
\text { B. } A_{3 \pi}
$$

Analogously, the formula for the $3 \pi$ asymmetry is

$$
A_{3 \pi}(t)=\frac{\operatorname{Tr}\left[O_{3 \pi} \bar{\rho}(t)\right]-\operatorname{Tr}\left[O_{3 \pi} \rho(t)\right]}{\operatorname{Tr}\left[O_{3 \pi} \bar{\rho}(t)\right]+\operatorname{Tr}\left[O_{3 \pi} \rho(t)\right]} ; \quad O_{3 \pi} \propto\left(\begin{array}{ll}
1 & 0 \\
0 & 0
\end{array}\right),
$$

from which one immediately obtains

$$
A_{3 \pi}(t)=\frac{\Delta \rho_{11}(t)}{\sum \rho_{11}(t)} .
$$

To first order in the small parameters, $\Delta \rho_{11}$ and $\Sigma \rho_{11}$ are given in Eqs. (46) and (49). This asymmetry can therefore be expressed as

$$
\begin{aligned}
A_{3 \pi}(t) & =\frac{2|\epsilon| \frac{\cos (\phi-\delta \phi)}{\cos \delta \phi}-\frac{2|\epsilon|}{\cos \delta \phi} e^{-1 / 2\left(\Gamma_{S}-\Gamma_{L}\right) t} \cos (\Delta m t+\phi-\delta \phi)}{1+\hat{\gamma}-\hat{\gamma} e^{-\left(\Gamma_{S}-\Gamma_{L}\right) t}} \\
& \approx[2|\epsilon| \cos \phi-4 \hat{\beta} \sin \phi \cos \phi]-2 e^{-1 / 2\left(\Gamma_{S}-\Gamma_{L}\right) t}\left[\operatorname{Re} \eta_{3 \pi} \cos \Delta m t-\operatorname{Im} \eta_{3 \pi} \sin \Delta m t\right],
\end{aligned}
$$

where, to facilitate contact with experiment, in the second form we have neglected the $\hat{\gamma}$ contribution, expressed $\delta \phi$ in terms of $\hat{\beta}$ (45), and defined

$$
\begin{gathered}
\operatorname{Re} \eta_{3 \pi}=|\epsilon| \cos \phi-2 \hat{\beta} \sin \phi \cos \phi, \\
\operatorname{Im} \eta_{3 \pi}=|\epsilon| \sin \phi+2 \hat{\beta} \cos ^{2} \phi .
\end{gathered}
$$

In the CPLEAR experiment, the time-dependent decay asymmetry into $\pi^{0} \pi^{+} \pi^{-}$is measured [3], and the data is fit to obtain the best values for $\operatorname{Re} \eta_{3 \pi}$ and $\operatorname{Im} \eta_{3 \pi}$. It would appear particularly useful to determine the ratio of these two parameters, so that a good fraction of the experimental un- certainties drops out. In the standard $C P$-violating scenario, the ratio is $\operatorname{Im} \eta_{3 \pi} / \operatorname{Re} \eta_{3 \pi}=\tan \phi \approx 1$, whereas in our scenario, it is

$$
\frac{\operatorname{Im} \eta_{3 \pi}}{\operatorname{Re} \eta_{3 \pi}}=\frac{|\epsilon| \sin \phi+2 \hat{\beta} \cos ^{2} \phi}{|\epsilon| \cos \phi-2 \hat{\beta} \sin \phi \cos \phi}=\tan (\phi-\delta \phi) .
$$

It is apparent from the above formulas that $A_{3 \pi}$ is much more sensitive to $\hat{\beta}$ than to $\hat{\alpha}$ or $\hat{\gamma}$. This sensitivity of $A_{3 \pi}$ to $\hat{\beta}$ is shown in Fig. 2(a), and that of $\left(\operatorname{Im} \eta_{3 \pi} / \operatorname{Re} \eta_{3 \pi}\right)$ in Fig. 2(b).

As already mentioned in Sec. II, additional information may be obtained from $\pi^{+} \pi^{-} \pi^{0}$ decays by observing the difference between the rates for $\pi^{+} \pi^{-} \pi^{0}$ decays with $m\left(\pi^{+} \pi^{0}\right)>m\left(\pi^{-} \pi^{0}\right)$ and $m\left(\pi^{+} \pi^{0}\right)<m\left(\pi^{-} \pi^{0}\right)$, [20] rep- 
resented by $O_{3 \pi}^{\text {int }}$ [Eqs. (19) and (20)]. This division of the final-state phase space into two halves is not $C P$ invariant, and hence enables one to measure interference between the $C P$-even $I_{3 \pi}=2$ and CP-odd $I_{3 \pi}=1$ final states. Defining

$$
A_{3 \pi}^{\text {int }}=\frac{\operatorname{Tr}\left[O_{3 \pi}^{\text {int }} \bar{\rho}(t)\right]-\operatorname{Tr}\left[O_{3 \pi}^{\text {int }} \rho(t)\right]}{\operatorname{Tr}\left[O_{3 \pi}^{\text {int }} \bar{\rho}(t)\right]+\operatorname{Tr}\left[O_{3 \pi}^{\text {int }} \rho(t)\right]},
$$

we obtain the formula

$$
A_{3 \pi}^{\text {int }}=\frac{\operatorname{Re} \Delta \rho_{12}}{\operatorname{Re} \Sigma \rho_{12}} .
$$

To first order in small parameters, we find

$$
A_{3 \pi}^{\mathrm{int}}=\frac{-e^{-\Gamma t}\left[\cos \Delta m t+\frac{2 \hat{\alpha}}{\tan \phi}[\sin \Delta m t-(\Delta m t) \cos \Delta m t]\right]}{\frac{|\epsilon|}{\cos \delta \phi}\left[\cos (\phi-\delta \phi) e^{\left.-\Gamma_{L^{t}}+\cos (\phi+\delta \phi) e^{-\Gamma_{S} t}-2 \cos \phi e^{-\Gamma t} \cos (\Delta m t-\delta \phi)\right]}\right.} .
$$

Note that $A_{3 \pi}^{\text {int }} \rightarrow \infty$ for $t \rightarrow 0$, signaling a breakdown of our approximation scheme. In the $C P T$-conserving case, this observable becomes

$$
A_{3 \pi}^{\text {int }} \rightarrow \frac{-e^{-\Gamma t} \cos \Delta m t}{|\epsilon| \cos \phi\left[e^{-\Gamma} L^{t}+e^{-\Gamma} S^{t}-2 e^{-\Gamma t} \cos \Delta m t\right]} .
$$

We see that this observable is sensitive to $\hat{\alpha}$ [see the numerator of (72)], and to $\hat{\beta}$ via $\delta \phi$. The sensitivity to $\hat{\alpha}$ may supplement usefully the information obtainable from the $A_{\Delta m}$ measurement discussed in Sec. IV E.

\section{C. $A_{\mathrm{T}}$}

The formula for this asymmetry, as obtained by applying the formalism of Sec. II, assumes the form

$$
A_{\mathrm{T}}=\frac{\Delta \rho_{11}+\Delta \rho_{22}+2 \operatorname{Re} \Sigma \rho_{12}}{\Sigma \rho_{11}+\Sigma \rho_{22}+2 \operatorname{Re} \Delta \rho_{12}}
$$

with the first-order expressions for $\Delta \rho_{11,22,12}$ and $\Sigma \rho_{11,22,12}$ given in Eqs. (46)-(51). In the usual non- $C P T$-violating case, one finds, to first order, the exactly time-independent result

$$
A_{\mathrm{T}}=4|\epsilon| \cos \phi
$$

as expected [3]. In the $C P T$-violating case, to first order, one finds a time-dependent expression

$$
A_{\mathrm{T}}=\frac{4|\epsilon|}{\cos \delta \phi}\left\{\frac{e^{-\Gamma_{L} t} \cos (\phi-\delta \phi)+e^{-\Gamma_{S} t} \cos (\phi+\delta \phi)-2 e^{-\Gamma t} \cos (\Delta m t-\delta \phi) \cos \phi}{e^{-\Gamma_{L} t}(1+2 \hat{\gamma})+e^{-\Gamma_{S} t}(1-2 \hat{\gamma})-2 e^{-\Gamma t}\left[\cos \Delta m t+\frac{2 \hat{\alpha}}{\tan \phi}(\sin \Delta m t-\Delta m t \cos \Delta m t)\right]}\right\}
$$

which asymptotes to

$$
A_{\mathrm{T}} \rightarrow \frac{4|\epsilon| \cos (\phi-\delta \phi)}{\cos \delta \phi(1+2 \hat{\gamma})}=\frac{4|\epsilon| \cos \phi-8 \hat{\beta} \sin \phi \cos \phi}{1+2 \hat{\gamma}}
$$

The sensitivities of $A_{\mathrm{T}}$ to $\hat{\alpha}$ and $\hat{\beta}$ are illustrated in Fig. 3(a) and 3(b), respectively. We see that the sensitivity to $\hat{\alpha}$ is again less than that to $\hat{\beta}$, and is restricted to $t / \tau_{s} \lesssim 15$, whereas the greater sensitivity to $\hat{\beta}$ persists to large $t$, as in Eq. (77), where the corresponding (utterly negligible) sensitivity to $\hat{\gamma}$ can be inferred.

\section{D. $A_{C P T}$}

Following the discussion in Sec. II, the formula for this observable, as defined by the CPLEAR Collaboration [3], is given by Eq. (22) with $f=\pi^{-} l^{+} \nu$ and $\bar{f}=\pi^{+} l^{-} \bar{\nu}$. We obtain

$$
A_{C P T}=\frac{\Delta \rho_{11}+\Delta \rho_{22}-2 \operatorname{Re} \Sigma \rho_{12}}{\sum \rho_{11}+\Sigma \rho_{22}-2 \operatorname{Re} \Delta \rho_{12}} .
$$

To first order, in both the $C P T$-conserving and $C P T$-violating cases, we find

$$
A_{C P T}=0 \text {. }
$$

To second order, the terms in the numerator of Eq. (78) can be written most succinctly in the long-time limit. With the help of the expressions in Appendix B, we obtain

$$
\begin{aligned}
& \Delta \rho_{11}^{(2)} \rightarrow-2|\epsilon| \hat{\gamma} \cos \phi+8|\epsilon| \hat{\alpha} \cos \phi \sin ^{2} \phi \\
&+4 \hat{\beta} \hat{\gamma} \sin \phi \cos \phi+16 \hat{\alpha} \hat{\beta} \sin \phi \cos ^{3} \phi \\
& \Delta \rho_{22}^{(2)} \rightarrow 2|\epsilon| \hat{\gamma} \cos \phi-4 \hat{\beta} \hat{\gamma} \sin \phi \cos \phi
\end{aligned}
$$




$$
\operatorname{Re} \Sigma \rho_{12}^{(2)} \rightarrow 4|\epsilon| \hat{\alpha} \cos \phi \sin ^{2} \phi+8 \hat{\alpha} \hat{\beta} \sin \phi \cos ^{3} \phi,
$$

which show that in the long-time limit, $A_{C P T}=0$ also to second order. In fact, some algebra shows that $A_{C P T}=0$ through second order for all values of $t$. This result implies that $\left|A_{C P T}\right| \lesssim 10^{-6}$ and thus is unobservably small.

We point out that this result is a quite distinctive signature of the modifications of the quantum mechanics proposed in Refs. $[6,15]$, since in the case of quantum-mechanical violation of $C P T$ symmetry [5], there is a nontrivial change in $A_{C P T}$, proportional to the $C P T$-violating parameters $\delta M$ and $\delta \Gamma$. Indeed, in Appendix A we obtain the following firstorder asymptotic result

$$
A_{C P T}^{\mathrm{QM}} \rightarrow 4 \sin \phi \cos \phi \widehat{\delta M}+2 \cos ^{2} \phi \widehat{\delta \Gamma},
$$

written in terms of the scaled variables. Part of the reason for this difference is the different role played by $\delta M$ as compared to the $\beta$ parameter in the formalism of Ref. [6], as discussed in detail in Ref. [15]. In particular, there are important sign differences between the ways that $\delta M$ and $\beta$ appear in the two formalisms, that cause the suppression to second order of any quantum-mechanical-violating effects in $A_{C P T}$, as opposed to the conventional quantum-mechanics case.

\section{E. $\boldsymbol{A}_{\Delta m}$}

Following Ref. [3], one can define $A_{\Delta m}$ as
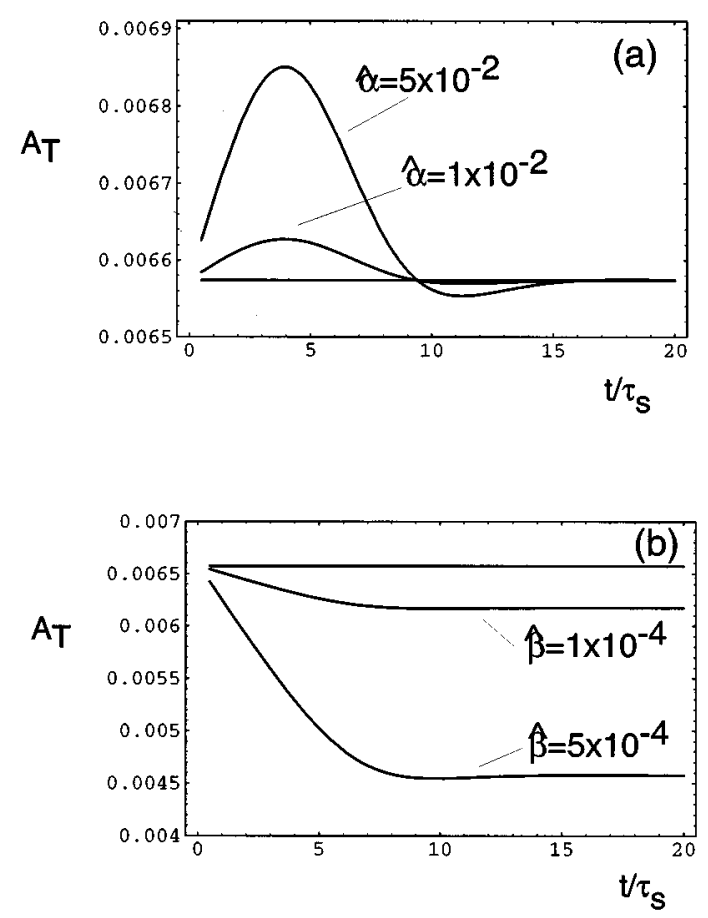

FIG. 3. The time-dependent asymmetry $A_{\mathrm{T}}$ for representative choices of (a) $\hat{\alpha}(\hat{\beta}=0)$ and (b) $\hat{\beta}(\hat{\alpha}=0)$. The dependence on $\hat{\gamma}$ is negligible. The flat line corresponds to the standard case.

$$
A_{\Delta m}=\frac{R\left(K^{0} \rightarrow \pi^{+}\right)+R\left(\bar{K}^{0} \rightarrow \pi^{-}\right)-R\left(\bar{K}^{0} \rightarrow \pi^{+}\right)-R\left(K^{0} \rightarrow \pi^{-}\right)}{R\left(K^{0} \rightarrow \pi^{+}\right)+R\left(\bar{K}^{0} \rightarrow \pi^{-}\right)+R\left(\bar{K}^{0} \rightarrow \pi^{+}\right)+R\left(K^{0} \rightarrow \pi^{-}\right)}
$$

in an obvious short-hand notation for the final states of the semileptonic decays, where only the pion content is shown explicitly. In the formalism of Sec. II, this expression becomes

$$
A_{\Delta m}=\frac{2 \operatorname{Re} \Delta \rho_{12}}{\Sigma \rho_{11}+\Sigma \rho_{22}} .
$$

The first-order expression in the usual nonCPT-violating case is

$$
A_{\Delta m}=-\frac{2 e^{-\Gamma t} \cos \Delta m t}{e^{-\Gamma_{L} t}+e^{-\Gamma} S^{t}},
$$

as obtained in Ref. [3]. In the $C P T$-violating case to first order, as Eqs. (48)-(50) show, neither $|\epsilon|$ nor $\hat{\beta}$ come in, and we obtain

$A_{\Delta m}=-\frac{2 e^{-\Gamma t}\left[\cos \Delta m t+\frac{2 \hat{\alpha}}{\tan \phi}(\sin \Delta m t-\Delta m t \cos \Delta m t)\right]}{e^{-\Gamma_{L} t}(1+2 \hat{\gamma})+e^{-\Gamma} S^{t}(1-2 \hat{\gamma})}$.

Since $\hat{\gamma}$ is negligible, this observable provides an exclusive test of $\hat{\alpha}$.
In the case of no $C P T$ violation, the observable $A_{\Delta m}$ has a minimum for $\tan \Delta m t=-\Gamma / \Delta m \approx-\frac{1}{2}|\Delta \Gamma| / \Delta m=$ $-1 / \tan \phi$. Since $\tan \phi \approx 1$, the minimum occurs for $\left(t / \tau_{s}\right)_{\min } \approx 3 \pi / 2$. In the $C P T$-violating case, Eq. (85) can be rewritten as

$$
A_{\Delta m}=-\frac{2 e^{-\Gamma t} C_{\Delta m} \cos \left(\Delta m t-\phi_{\Delta m}\right)}{e^{-\Gamma_{L} t}(1+2 \hat{\gamma})+e^{-\Gamma} S^{t}(1-2 \hat{\gamma})},
$$

with

$$
\tan \phi_{\Delta m}=\frac{2 \hat{\alpha} / \tan \phi}{1-\hat{\alpha} t|\Delta \Gamma|}, \quad C_{\Delta m}=\frac{1-\hat{\alpha} t|\Delta \Gamma|}{\cos \phi_{\Delta m}}
$$

Since the minimum occurs for $t|\Delta \Gamma| \sim 5$, for small values of $\hat{\alpha}$, one can neglect the time-dependent pieces in $\phi_{\Delta m}$ and $C_{\Delta m}$. The new minimum condition for $A_{\Delta m}$ is then modified to $\tan \left(\Delta m t-\phi_{\Delta m}\right) \approx-1 / \tan \phi$, and thus the minimum is shifted to

$$
\left(t / \tau_{s}\right)_{\min } \approx \frac{3 \pi}{2}+4 \hat{\alpha}
$$



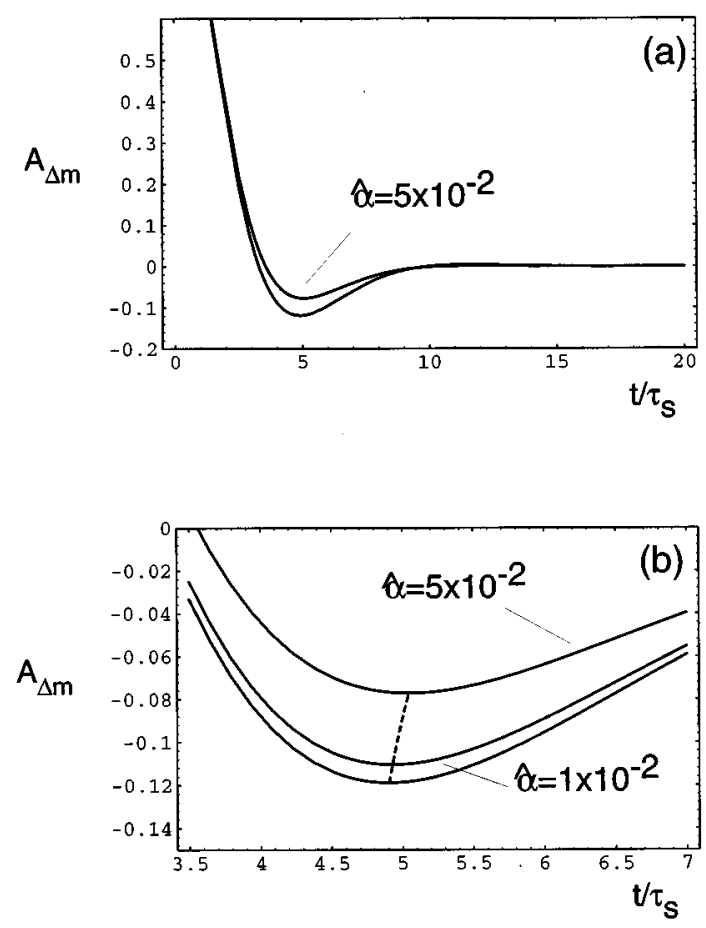

FIG. 4. The time-dependent asymmetry $A_{\Delta m}$ for representative choices of $\hat{\alpha}(\hat{\beta}=\hat{\gamma}=0)$. This asymmetry depends most sensitively only on $\hat{\alpha}$. In both panels, the bottom curve corresponds to the standard case. In the detail (b), the dashed line indicates the location of the minimum as $\hat{\alpha}$ is varied.

for small values of $\hat{\alpha}$. A similar test for $\hat{\alpha}$ was proposed in Ref. [16], where it was based on the traditional semileptonic decay charge asymmetry parameter $\delta(t)[15]$. However, to first order that observable depends also on $|\epsilon|$ and $\hat{\beta}$, and as such it is not a direct test of $\hat{\alpha}$, as opposed to the one proposed here. Figure 4 exhibits the sensitivity of $A_{\Delta m}$ to $\hat{\alpha}$, including (a) the general dependence in the interference region and (b) the detailed location of the minimum as $\hat{\alpha}$ is varied.

\section{REGENERATION}

\section{A. Simplified thin-regenerator case}

Regeneration involves the coherent scattering of a $K^{0}$ or $\bar{K}^{0}$ off a nuclear target, which we assume can be described using the normal framework of quantum field theory and quantum mechanics. Thus we describe it by an effective Hamiltonian which takes the form

$$
\Delta H=\left(\begin{array}{cc}
T & 0 \\
0 & \bar{T}
\end{array}\right)
$$

in the $\left(K^{0}, \bar{K}^{0}\right)$ basis, where

$$
T=\frac{2 \pi N}{m_{K}} \mathscr{C l}, \quad \bar{T}=\frac{2 \pi N}{m_{K}} \overline{\mathscr{C}},
$$

with $\mathscr{C}=\left\langle K^{0}|A| K^{0}\right\rangle$ the forward $K^{0}$-nucleus scattering amplitude (and analogously for $\overline{\mathscr{C}}$ ), and $N$ is the nuclear regenerator density. We can rewrite $\Delta H(89)$ in the $K_{1,2}$ basis as

$$
\Delta H=\left(\begin{array}{cc}
T+\bar{T} & T-\bar{T} \\
T-\bar{T} & T+\bar{T}
\end{array}\right)
$$

which can, in principle, be included as a contribution to $H$ in the density-matrix equation:

$$
\partial_{t} \rho=-i[H, \rho]+i \delta H \rho,
$$

where $\delta H$ represents the possible $C P T$ - and quantummechanical $(\mathrm{QM})$-violating term.

It may be adequate as a first approximation to treat the regenerator as very thin, in which case we may use the impulse approximation, and the regenerator changes $\rho$ by an amount

$$
\delta \rho=-i[\Delta \mathscr{H}, \rho],
$$

where

$$
\Delta \mathscr{H}=\int d t \Delta H .
$$

Writing

$$
\rho=\left(\begin{array}{ll}
\rho_{11} & \rho_{12}^{*} \\
\rho_{12} & \rho_{22}
\end{array}\right),
$$

in this approximation, we obtain

$$
\delta \rho=-i \Delta T\left(\begin{array}{cc}
2 i \operatorname{Im} \rho_{12} & -\rho_{11}+\rho_{22} \\
\rho_{11}-\rho_{22} & -2 i \operatorname{Im} \rho_{12}
\end{array}\right),
$$

where

$$
\Delta T \equiv \int d t(T-\bar{T}) .
$$

This change in $\rho$ enables the possible $C P T$ - and QMviolating terms in (92) to be probed in a new way. Consider the idealization that the neutral $K$ beam is already in a $K_{L}$ state [Eq. (31)]:

$$
\rho=\rho_{L} \approx\left(\begin{array}{cc}
1 & \epsilon^{*}+B^{*} \\
\epsilon+B & |\epsilon|^{2}+C
\end{array}\right),
$$

where

$$
B=-i 2 \hat{\beta} \cos \phi e^{-i \phi}, \quad C=\hat{\gamma}-4 \hat{\beta}^{2} \cos ^{2} \phi-4 \hat{\beta}|\epsilon| \sin \phi .
$$

Substituting Eqs. (98) and (99) into Eq. (96), we find that in the joint large- $t$ and impulse approximations 


$$
\rho+\delta \rho=\left(\begin{array}{cc}
1+2 \Delta T \operatorname{Im}(\epsilon+B) & \epsilon^{*}+B^{*}+i\left(1-|\epsilon|^{2}-C\right) \Delta T \\
\epsilon+B-i\left(1-|\epsilon|^{2}-C\right) \Delta T & |\epsilon|^{2}+C-2 \Delta T \operatorname{Im}(\epsilon+B)
\end{array}\right)
$$

We see that the usual semileptonic decay asymmetry observable

$$
O_{\pi^{-} l^{+} \nu}-O_{\pi^{+} l^{-} \bar{\nu}}=\left(\begin{array}{cc}
0 & 2 \\
2 & 0
\end{array}\right)
$$

which measures $\operatorname{Re}(\epsilon+B)$ in the case without the regenerator, receives no contribution from the regenerator (i.e., $\Delta T$ cancels out in the sum of the off-diagonal elements). On the other hand, there is a new contribution to the value of $R_{2 \pi}=R\left(K_{L} \rightarrow 2 \pi\right)=\operatorname{Tr}\left[O_{2 \pi} \rho\right]=\rho_{22}$ : namely,

$$
R_{2 \pi}=|\epsilon|^{2}+\hat{\gamma}-4 \hat{\beta}^{2} \cos ^{2} \phi-4 \hat{\beta}|\epsilon| \sin \phi-2 \Delta T \operatorname{Im}(\epsilon+B) .
$$

The quantity $\operatorname{Im}(\epsilon+B)$ was not accessible directly to the observable $R_{2 \pi}$ in the absence of a regenerator. Theoretically, the phases of $\epsilon$ and $B$ (99) are fixed: i.e.,

$$
\operatorname{Im}(\epsilon+B)=-|\epsilon| \frac{\sin (\phi-\delta \phi)}{\cos \delta \phi}=-|\epsilon| \sin \phi-2 \hat{\beta} \cos ^{2} \phi .
$$

Nevertheless, this phase prediction should be checked, so the regenerator makes a useful addition to the physics program.

The above analysis is oversimplified, since the impulse approximation may not be sufficiently precise, and the neutral $K$ beam is not exactly in a $K_{L}$ state. Moreover, the result in Eq. (100) is valid only at the time the beam emerges from the regenerator. However, this simple example may serve to illustrate the physics interest of measurements using a regenerator. We next generalize the analysis to include a general neutral $K$ beam encountering a thin regenerator, with the full time dependence after leaving the regenerator.

\section{B. Detailed regenerator tests}

To make contact with the overall discussion in this paper, we envision the following scenario.

(i) Pure $K^{0}, \bar{K}^{0}$ beams are produced at time $t=0$, corresponding to initial density matrices $\rho_{0}$ and $\bar{\rho}_{0}$, respectively.

(ii) These beams are described by density matrices $\rho(t)$ and $\bar{\rho}(t)$, and evolve with time as described in Sec. II, until a time $t=t_{r}$ where they are described by $\rho\left(t_{r}\right)$ and $\bar{\rho}\left(t_{r}\right)$.

(iii) At $t=t_{r}$, a thin regenerator is encountered. ${ }^{3}$ In our thin-regenerator approximation (described in the previous subsection), at $t=t_{r}$, suddenly the density matrices receive an additional contribution $\delta \rho\left(t_{r}\right)$ or $\delta \bar{\rho}\left(t_{r}\right)$, according to Eq. (96).

\footnotetext{
${ }^{3}$ For simplicity, we assume that the regenerator is encountered at the same $\Delta t=t_{r}$ after production for all beam particles. In specific experimental setups, our expressions would need to be folded with appropriate geometrical functions.
}

(iv) For $\tau=t-t_{r} \geqslant 0$, the beams are described by density matrices $\rho^{r}(\tau)$ and $\bar{\rho}^{r}(\tau)$, which again evolve as described in Sec. II, but this time with initial conditions $\rho^{r}(0)=\rho\left(t_{r}\right)+\delta \rho\left(t_{r}\right)$ and $\bar{\rho}^{r}(0)=\bar{\rho}\left(t_{r}\right)+\delta \bar{\rho}\left(t_{r}\right)$.

In this context, we consider two kinds of tests. In a CPLEAR-like scenario, the identity of the beam is known irrespective of the presence of the regenerator, and thus a measurement of $A_{2 \pi}^{r}(\tau)$, i.e., $A_{2 \pi}$ after the thin regenerator is traversed, appears feasible. The second test is reminiscent of the Fermilab experiments, where the experimental setup is such that $t_{r} \gg \tau_{S}$, and the beam is in a $K_{L}$ state. After the regenerator is traversed, one then measures $R_{2 \pi}$ in the interference region.

Before embarking on elaborate calculations, we should perhaps quantify our "thin-regenerator" criterion. For the impulse approximation to be valid, $\delta \rho$ in Eq. (96) should not change $\rho$ by too much. Since the entries in $\rho$ are typically $\sim 10^{-3}$ or smaller, we should demand that $\Delta T$ be a reasonably small number. Let us estimate $\Delta T=\int d t(T-\bar{T})$. Assuming $\mathscr{C}-\overline{\mathscr{C}} \boldsymbol{b} \sim 1 / m_{\pi}$ and relativistic kaons, we obtain

$$
\Delta T \approx \frac{1}{30} \text { thickness }[\mathrm{cm}] \text { density }\left[\mathrm{g} / \mathrm{cm}^{3}\right],
$$

and thus a "thin" regenerator should have a thickness $\lesssim 1 \mathrm{~cm}$. This estimate appears reasonable when considering that in the $2 \mathrm{~ns}$ or so that the beams are usually observed (about $20 \tau_{S}$ ), they travel $\sim 60 \mathrm{~cm}$. Such a regenerator could conceivably be installed in an upgraded CPLEAR experiment. In the Fermilab E731 [21] and E773 [22,23] experiments, the regenerators used are much thicker, and the validity of our approximation is unclear.

$$
\text { 1. } A_{2 \pi}^{r}
$$

We start with $A_{2 \pi}^{r}=\Delta \rho_{22}^{r} / \Sigma \rho_{22}^{r}$, where, e.g., $\Delta \rho_{22}^{r}(\tau)$ is given by $\rho_{22}(\tau)$ in Eqs. (37) and (43) with $\rho(0) \rightarrow \Delta \rho^{r}(0)=\Delta \rho\left(t_{r}\right)+\delta(\Delta \rho)\left(t_{r}\right)$, and $\delta(\Delta \rho)$ given in Eq. (96) with $\rho \rightarrow \Delta \rho$. We obtain

$$
\begin{aligned}
\Delta \rho_{22}^{r}(\tau)= & \left\{\left[\Delta \rho_{22}\left(t_{r}\right)-2 \Delta T \operatorname{Im} \Delta \rho_{12}\left(t_{r}\right)\right]-\hat{\gamma}\left[\Delta \rho_{11}\left(t_{r}\right)\right.\right. \\
& \left.+2 \Delta T \operatorname{Im} \Delta \rho_{12}\left(t_{r}\right)\right] \\
& \left.-2|\epsilon| \frac{\cos \left(\phi+\delta \phi+\Delta \phi_{12}\right)}{\cos \delta \phi}\left|\Delta \rho_{12}^{r}(0)\right|\right\} \\
& \times e^{-\Gamma_{S} \tau}+\hat{\gamma}\left[\Delta \rho_{11}\left(t_{r}\right)+2 \Delta T \operatorname{Im} \Delta \rho_{12}\left(t_{r}\right)\right] \\
& \times e^{-\Gamma_{L} \tau}+\frac{2|\epsilon|}{\cos \delta \phi}\left|\Delta \rho_{12}^{r}(0)\right| e^{-\Gamma \tau} \cos \\
& \times\left(\Delta m \tau-\phi-\delta \phi-\Delta \phi_{12}\right)
\end{aligned}
$$




$$
\begin{aligned}
\Sigma \rho_{22}^{r}(\tau)= & \left\{\left[\Sigma \rho_{22}\left(t_{r}\right)-2 \Delta T \operatorname{Im} \Sigma \rho_{12}\left(t_{r}\right)\right]-\hat{\gamma}\left[\Sigma \rho_{11}\left(t_{r}\right)\right.\right. \\
& \left.+2 \Delta T \operatorname{Im} \Sigma \rho_{12}\left(t_{r}\right)\right] \\
& \left.-2|\epsilon| \frac{\cos \left(\phi+\delta \phi+\Sigma \phi_{12}\right)}{\cos \delta \phi}\left|\Sigma \rho_{12}^{r}(0)\right|\right\} e^{-\Gamma_{S} \tau} \\
& +\hat{\gamma}\left[\Sigma \rho_{11}\left(t_{r}\right)+2 \Delta T \operatorname{Im} \Sigma \rho_{12}\left(t_{r}\right)\right] e^{-\Gamma_{L} \tau} \\
& +\frac{2|\epsilon|}{\cos \delta \phi}\left|\Sigma \rho_{12}^{r}(0)\right| e^{-\Gamma \tau} \cos (\Delta m \tau-\phi-\delta \phi \\
& \left.-\Sigma \phi_{12}\right),
\end{aligned}
$$

where we have defined the phases $\Delta \phi_{12}$ and $\Sigma \phi_{12}$ through

$$
\begin{aligned}
& \Delta \rho_{12}^{r}(0)=\left|\Delta \rho_{12}\left(t_{r}\right)-i \Delta T\left[\Delta \rho_{11}\left(t_{r}\right)-\Delta \rho_{22}\left(t_{r}\right)\right]\right| e^{i \Delta \phi_{12}} \\
& \Sigma \rho_{12}^{r}(0)=\left|\Sigma \rho_{12}\left(t_{r}\right)-i \Delta T\left[\Sigma \rho_{11}\left(t_{r}\right)-\Sigma \rho_{22}\left(t_{r}\right)\right]\right| e^{i \Sigma \phi_{12}}
\end{aligned}
$$

In these expressions, the "initial-condition" input matrices $\Delta \rho\left(t_{r}\right)$ and $\Sigma \rho\left(t_{r}\right)$ are obtained from Eqs. (46)-(51) by inserting $t=t_{r}$. We obtain a rather complicated result, which, in addition to the $C P T$-violating parameters, also depends on $\Delta T$ and $t_{r}$. To illustrate the behavior of $A_{2 \pi}^{r}$, let us consider two limiting cases: $t_{r} \ll \tau_{S}$ and $t_{r} \gg \tau_{S}$. For a regenerator very close to the origin $\left(t_{r} \ll \tau_{S}\right)$, we basically have $\Delta \rho\left(t_{r}\right) \approx \Delta \rho(0)$ and $\Sigma \rho\left(t_{r}\right) \approx \Sigma \rho(0)$, as in Eq. (28), and we obtain

$$
\begin{gathered}
\Delta \rho_{22}^{r}(\tau) \rightarrow 2|\epsilon| \frac{\cos (\phi+\delta \phi)}{\cos \delta \phi} e^{-\Gamma_{S} \tau} \\
-\frac{2|\epsilon|}{\cos \delta \phi} e^{-\Gamma \tau} \cos (\Delta m \tau-\phi-\delta \phi), \\
\Sigma \rho_{22}^{r}(\tau) \rightarrow(1-\hat{\gamma}) e^{-\Gamma_{S} \tau}+\hat{\gamma} e^{-\Gamma_{L} \tau} .
\end{gathered}
$$

Neglecting $\hat{\gamma}$, we find

$$
\begin{aligned}
A_{2 \pi}^{r}(\tau) \rightarrow & \frac{2|\epsilon|}{\cos \delta \phi}\{\cos (\phi+\delta \phi) \\
& \left.-e^{1 / 2\left(\Gamma_{S}-\Gamma_{L}\right) \tau} \cos (\Delta m \tau-\phi-\delta \phi)\right\}
\end{aligned}
$$

Thus, when the regenerator is placed near the production point, the effects of $\Delta T$ drop out, and the result without a regenerator is recovered [see Eq. (53) dropping $\hat{\gamma}$ and all second-order terms].

Of more interest is the case of a regenerator placed in the asymptotic region $\left(t_{r} \gg \tau_{S}\right)$. In this case, the expressions for $\Delta \rho\left(t_{r}\right)$ and $\Sigma \rho\left(t_{r}\right)$ simplify considerably, through first order:

$$
\begin{gathered}
\Delta \rho_{11}\left(t_{r}\right) \rightarrow \frac{2|\epsilon| \cos (\phi-\delta \phi)}{\cos \delta \phi} \quad \Sigma \rho_{11}\left(t_{r}\right) \rightarrow 1+\hat{\gamma} \approx 1, \\
\Delta \rho_{22}\left(t_{r}\right) \rightarrow 0 \quad \Sigma \rho_{22}\left(t_{r}\right) \rightarrow \hat{\gamma} \approx 0, \\
\Delta \rho_{12}\left(t_{r}\right) \rightarrow 0 \quad \Sigma \rho_{12}\left(t_{r}\right) \rightarrow \frac{|\epsilon|}{\cos \delta \phi} e^{i(\delta \phi-\phi)} .
\end{gathered}
$$

Inserting these limiting expressions (and taking $\hat{\gamma}=0$ ), we obtain

$$
\begin{aligned}
\Delta \rho_{22}^{r}(\tau) \rightarrow & -2|\epsilon| \frac{\cos \left(\phi+\delta \phi+\Delta \phi_{12}\right)}{\cos \delta \phi}\left|\Delta \rho_{12}^{r}(0)\right| e^{-\Gamma_{S} \tau} \\
& +\frac{2|\epsilon|}{\cos \delta \phi}\left|\Delta \rho_{12}^{r}(0)\right| e^{-\Gamma \tau} \\
& \times \cos \left(\Delta m \tau-\phi-\delta \phi-\Delta \phi_{12}\right) \\
\Sigma \rho_{22}^{r}(\tau) \rightarrow & \left\{2 \Delta T|\epsilon| \frac{\sin (\phi-\delta \phi)}{\cos \delta \phi}\right. \\
& \left.-2|\epsilon| \frac{\cos \left(\phi+\delta \phi+\Sigma \phi_{12}\right)}{\cos \delta \phi}\left|\Sigma \rho_{12}^{r}(0)\right|\right\} e^{-\Gamma_{S} \tau} \\
& +\frac{2|\epsilon|}{\cos \delta \phi}\left|\Sigma \rho_{12}^{r}(0)\right| e^{-\Gamma \tau} \\
& \times \cos \left(\Delta m \tau-\phi-\delta \phi-\Sigma \phi_{12}\right)
\end{aligned}
$$

and thus
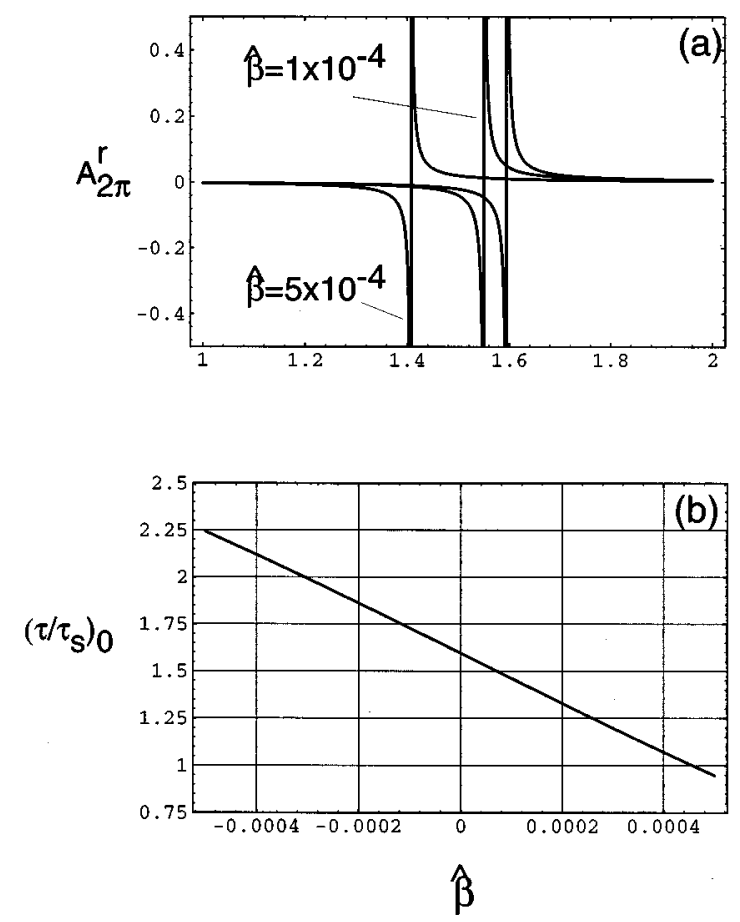

FIG. 5. The time-dependent asymmetry $A_{2 \pi}^{r}(\tau)$ in the presence of a thin regenerator placed far from the production point, as a function of the time $\tau$ after leaving the regenerator, for representative choices of $\hat{\beta}\left[A_{2 \pi}^{r}(\tau)\right.$ is rather insensitive to $\hat{\alpha}, \hat{\gamma}$, which are set to zero]. The right-most curve corresponds to the standard case. Also shown (b) is the position of the (first) zero in $A_{2 \pi}^{r}(\tau)$ as a function of $\hat{\beta}$. 


$$
\left.A_{2 \pi}^{r}(\tau) \rightarrow \frac{\left|\Delta \rho_{12}^{r}(0)\right|}{\left|\Sigma \rho_{12}^{r}(0)\right|} \frac{-\cos \left(\phi+\delta \phi+\Delta \phi_{12}\right)+e^{1 / 2\left(\Gamma_{S}-\Gamma_{L}\right) \tau} \cos \left(\Delta m \tau-\phi-\delta \phi-\Delta \phi_{12}\right)}{\left[\frac{\Delta T}{\left|\Sigma \rho_{12}^{r}(0)\right|} \sin (\phi-\delta \phi)-\cos \left(\phi+\delta \phi+\Sigma \phi_{12}\right)\right]+e^{1 / 2\left(\Gamma_{S}-\Gamma_{L}\right) \tau} \cos (\Delta m \tau-\phi-\delta \phi-\Sigma \phi}\right)
$$

with

$$
\begin{gathered}
\Delta \rho_{12}^{r}(0) \rightarrow\left|-i \Delta T \frac{2|\epsilon| \cos (\phi-\delta \phi)}{\cos \delta \phi}\right| e^{i \Delta \phi_{12} \Rightarrow \Delta \phi_{12}}=-\frac{\pi}{2}, \\
\Sigma \rho_{12}^{r}(0) \rightarrow\left|\frac{|\epsilon|}{\cos \delta \phi} e^{i(\delta \phi-\phi)}-i \Delta T\right| e^{i \Sigma \phi_{12}} .
\end{gathered}
$$

The result in Eq. (115) reveals a large shift $\left(\Delta \phi_{12}=-\frac{\pi}{2}\right)$ in the interference pattern relative to the case of no regenerator. According to our estimate of $\Delta T$ in Eq. (104), it would appear that $\Delta T \gg|\epsilon|$ is a case of interest to consider. In this limit, $\Delta T$ drops out from the $A_{2 \pi}^{r}$ observable, $\Delta \phi_{12}=\Sigma \phi_{12}=-\frac{\pi}{2}$, and

$$
A_{2 \pi}^{r}(\tau) \rightarrow 2|\epsilon| \frac{\cos (\phi-\delta \phi)}{\cos \delta \phi} \frac{\sin (\phi+\delta \phi)+e^{1 / 2\left(\Gamma_{S}-\Gamma_{L}\right) \tau} \sin (\Delta m \tau-\phi-\delta \phi)}{\sin (\phi+\delta \phi)-\sin (\phi-\delta \phi)+e^{1 / 2\left(\Gamma_{S}-\Gamma_{L}\right) \tau} \sin (\Delta m \tau-\phi-\delta \phi)} .
$$

The time dependence of $A_{2 \pi}^{r}(\tau)$ is shown in Fig. 5 from which it is apparent that $A_{2 \pi}^{r}(\tau)$ is basically flat except for values of $\tau$ for which $\sin (\Delta m \tau-\phi-\delta \phi)=0$. This occurs for $\left(\tau / \tau_{S}\right)_{0} \approx 2\left(n \pi+\frac{\pi}{4}+\delta \phi\right)$, a result which is plotted against $\hat{\beta}$ (for $n=0$ ) also in Fig. 5. We note that for increasingly larger values of $n$, the structure in the curves becomes narrower and narrower and therefore much less sensitive to $\hat{\beta}$, with the first zero $(n=0)$ possibly being the only observable one.

\section{2. $R_{2 \pi}$}

The observable $R_{2 \pi}=R(K \rightarrow 2 \pi)$ has traditionally been the focus of $C P$-violation studies. Because the detector is physically located a distance away from the source of the neutral kaons, most of the $K_{S}$ component of the beam decays away, and one is basically sensitive only to the $K_{L} \rightarrow 2 \pi$ decays. To study also the interesting interference region, a regenerator is inserted in the path of the $K_{L}$ particles right before they reach the detector, so that $K_{S}$ particles are regenerated and interference studies are possible. Unfortunately, the regenerator complicates the physics somewhat. To simplify the problem, let us first consider the case of a pure $K^{0}$ beam whose decay products can be detected from the instant of production (not unlike in the CPLEAR experiment). We will address the effect of the regenerator in the next subsection.

In our formalism, the $R_{2 \pi}$ observable corresponds to the operator $O_{2 \pi}$ in (17), which gives

$$
R_{2 \pi}(t)=\rho_{22}(t)
$$

Through second order, the corresponding expression is obtained from Eqs. (37), (43), and (B16) by inserting $\rho_{11}(0)=\rho_{22}(0)=\rho_{12}(0)=1$. In the case of standard quantum-mechanical $C P$ violation, one obtains

$$
R_{2 \pi}(t)=c_{S} e^{-\Gamma_{S} t}+c_{L} e^{-\Gamma_{L} t}+2 c_{I} e^{-\Gamma t} \cos (\Delta m t-\phi),
$$

where to second order, the $c_{S}, c_{L}, c_{I}$ coefficients are given by:

$$
\begin{gathered}
c_{S}=1-2|\epsilon| \cos \phi+|\epsilon|^{2}(1+2 \cos 2 \phi+t|\Delta \Gamma|), \\
c_{L}=|\epsilon|^{2} \\
c_{I}=|\epsilon|-2|\epsilon|^{2} \cos \phi .
\end{gathered}
$$

It is then apparent that to the order calculated: $c_{I}^{2}=c_{S} c_{L}=|\epsilon|^{2}$. Violations of this relation would indicate departures from standard quantum mechanics, which can be parametrized by [24]

$$
\zeta=1-\frac{c_{I}}{\sqrt{c_{S} c_{L}}}
$$

In our quantum-mechanical-violating framework, we expect $\zeta \neq 0$. Indeed, we obtain

$$
\begin{gathered}
c_{S}=1-\hat{\gamma}-2|\epsilon| \frac{\cos (\phi+\delta \phi)}{\cos \delta \phi} \\
c_{L}=\hat{\gamma}+\hat{\gamma}^{2}+|\epsilon|^{2} \frac{\cos (\phi-2 \delta \phi)}{\cos \phi \cos ^{2} \delta \phi}-2|\epsilon| \hat{\gamma} \frac{\cos (\phi-\delta \phi)}{\cos \delta \phi} \\
c_{I}=\frac{|\epsilon|}{\cos \delta \phi}
\end{gathered}
$$

where only terms relevant to the computation of $\zeta$ to second order have been kept (note that $\hat{\alpha}$ does not contribute to $\zeta$ to the order calculated). Also, in this case the general relation in 
Eq. (120) gets modified by a phase shift in the interference term $\phi \rightarrow \phi+\delta \phi$. Using these expressions, we obtain ${ }^{4}$

$$
\begin{aligned}
\frac{c_{I}^{2}}{c_{S} c_{L}} & =\frac{|\epsilon|^{2} / \cos ^{2} \delta \phi}{\hat{\gamma}(1-4|\epsilon| \cos \phi)+|\epsilon|^{2} \frac{\cos (\phi-2 \delta \phi)}{\cos \phi \cos ^{2} \delta \phi}} \\
& \approx \frac{1}{\frac{\hat{\gamma}}{|\epsilon|^{2}}+\frac{\cos (\phi-2 \delta \phi)}{\cos \phi}}
\end{aligned}
$$

and thus

$$
\zeta \approx \frac{1}{2}\left[1-\frac{1}{\frac{\hat{\gamma}}{|\epsilon|^{2}}+\frac{\cos (\phi-2 \delta \phi)}{\cos \phi}}\right] \approx \frac{\hat{\gamma}}{2|\epsilon|^{2}}-\frac{2 \hat{\beta}}{|\epsilon|} \sin \phi,
$$

where the second form holds for small values of $\hat{\gamma} /|\epsilon|^{2}$ and $\delta \phi \approx-2 \hat{\beta} \cos \phi /|\epsilon|$. The parameter $\zeta$ has been measured to be $\zeta^{\exp }=0.03 \pm 0.02 \quad$ [25]. Setting $\hat{\beta}=0$, one obtains $\hat{\gamma} \approx(3 \pm 2) \times 10^{-7}$ [24]. More generally, the dependence of $\zeta$ on $\hat{\beta}$ and $\hat{\gamma}$ is shown in Fig. 6, along with the present experimental limits on $\zeta$.

$$
\rho\left(t_{r}\right)=\left(\begin{array}{cc}
1+\hat{\gamma}-2|\epsilon| \cos \phi+4 \hat{\beta} \sin \phi \cos \phi & (|\epsilon|+i 2 \hat{\beta} \cos \phi) e^{i \phi} \\
(|\epsilon|-i 2 \hat{\beta} \cos \phi) e^{-i \phi} & |\epsilon|^{2}+\hat{\gamma}-4 \hat{\beta}^{2} \cos ^{2} \phi-4 \hat{\beta}|\epsilon| \sin \phi
\end{array}\right) .
$$

Note that at the instant the beam leaves the regenerator $(\tau=0), \quad$ we $\quad$ obtain $\quad R_{2 \pi}^{r}(0)=\rho_{22}^{r}(0)=\rho_{22}\left(t_{r}\right)$ $-2 \Delta T \operatorname{Im} \rho_{12}\left(t_{r}\right)$ which, after inserting $\rho\left(t_{r}\right)$ from Eq. (132), agrees with the result derived above in Eq. (102) where no time dependence after leaving the regenerator was considered.

In the interference region, the expression for $R_{2 \pi}$ simplifies considerably: we keep only the term proportional to $e^{-\Gamma \tau}$

$$
R_{2 \pi}^{\mathrm{int}}(\tau)=\frac{2|\epsilon|}{\cos \delta \phi}\left|\rho_{12}^{r}(0)\right| e^{-\Gamma \tau} \cos \left(\Delta m \tau-\phi-\delta \phi-\phi_{12}\right),
$$

with

$$
\rho_{12}^{r}(0) \approx\left|\frac{|\epsilon|}{\cos \delta \phi} e^{i(\delta \phi-\phi)}-i \Delta T\right| e^{i \phi_{12}} .
$$

\footnotetext{
${ }^{4}$ Note that in the scenario discussed in Sec. IV A, where $C P T$ violation accounts for the observed $C P$ violation (i.e., $|\epsilon|=0$, $\left.2 \hat{\beta} \cos \phi \rightarrow \pm|\epsilon|, \quad \hat{\gamma} \rightarrow 2|\epsilon|^{2}\right)$, one obtains $\quad c_{I}^{2} /\left(c_{S} c_{L}\right) \rightarrow 1 \Leftrightarrow \zeta=0$. (This result was implicitly obtained in Ref. [15].) Such result is not enough to validate the scenario, since as discussed above, this scenario is fatally flawed by the large phase shift in the interference term.
}

Let us now turn to the $R_{2 \pi}^{r}=\rho_{22}^{r}(\tau)$ observable in the presence of a thin regenerator. Here, $\rho_{22}^{r}(\tau)$ is given to first order by Eqs. (37) and (43) with $\rho(0) \rightarrow \rho^{r}(0)=\rho\left(t_{r}\right)$ $+\delta \rho\left(t_{r}\right)$, and $\delta \rho$ given in Eq. (96). We obtain

$$
\begin{aligned}
R_{2 \pi}^{r}(\tau)= & \left\{\left[\rho_{22}\left(t_{r}\right)-2 \Delta T \operatorname{Im} \rho_{12}\left(t_{r}\right)\right]-\hat{\gamma}\left[\rho_{11}\left(t_{r}\right)\right.\right. \\
& \left.+2 \Delta T \operatorname{Im} \rho_{12}\left(t_{r}\right)\right] \\
& \left.-2|\epsilon| \frac{\cos \left(\phi+\delta \phi+\phi_{12}\right)}{\cos \delta \phi}\left|\rho_{12}^{r}(0)\right|\right\} e^{-\Gamma_{S} \tau} \\
& +\hat{\gamma}\left[\rho_{11}\left(t_{r}\right)+2 \Delta T \operatorname{Im} \rho_{12}\left(t_{r}\right)\right] e^{-\Gamma_{L} \tau} \\
& +\frac{2|\epsilon|}{\cos \delta \phi}\left|\rho_{12}^{r}(0)\right| e^{-\Gamma \tau} \cos \left(\Delta m \tau-\phi-\delta \phi-\phi_{12}\right),
\end{aligned}
$$

where

$$
\rho_{12}^{r}(0)=\left|\rho_{12}\left(t_{r}\right)-i \Delta T\left[\rho_{11}\left(t_{r}\right)-\rho_{22}\left(t_{r}\right)\right]\right| e^{i \phi_{12}} .
$$

As we discussed above, the initial condition matrix $\rho\left(t_{r}\right)$ is simply $\rho_{L}$ : namely,
In this case, we again see that the regenerator introduces a shift in the interference pattern and modifies its overall magnitude, even in the absence of $C P T$ violation. In the limit in which $\Delta T \gg|\epsilon|,\left|\rho_{12}^{r}(0)\right| \rightarrow \Delta T, \phi_{12} \rightarrow-\frac{\pi}{2}$ and

$$
R_{2 \pi}^{\mathrm{int}}(\tau) \rightarrow \frac{2|\epsilon| \Delta T}{\cos \delta \phi} e^{-\Gamma \tau} \cos \left(\Delta m \tau-\phi-\delta \phi+\frac{\pi}{2}\right),
$$

which exhibits a large phase shift and a distinctive linear dependence on $\Delta T$, it is a nice signature. Moreover, the result still allows a determination of the $C P T$-violating parameter $\beta$, through $\delta \phi(45)$.

We now address the $\zeta$ parameter in the presence of a regenerator. Let us first start with the case of standard quantum mechanics, where we expect $\zeta$ to vanish. Looking back at Eqs. (121)-(123), we see that (to the order calculated) the $\zeta=0$ relation amounts to $\left[c_{I}^{(1)}\right]^{2}=c_{S}^{(0)} c_{2}^{(2)}$, where the orders at which the relevant contributions appear have been indicated. In the case of a regenerator, the time dependence of $\rho_{22}^{r}(\tau)$ is the same as that of $\rho_{22}(t)$, the only difference being in the coefficients which depend on different initial-condition matrices $\left[\rho^{r}(0)\right.$ vs $\left.\rho(0)\right]$. To make our result more general, we will keep this initial-condition matrix unspecified. Using Eqs. (37), (43), and (B16), we then get 


$$
\begin{gathered}
c_{S}^{(0)}=\rho_{22}(0), \\
c_{L}^{(2)}=\rho_{11}(0)|\epsilon|^{2}, \\
c_{I}^{(1)}=\left|\rho_{12}(0)\right||\epsilon|,
\end{gathered}
$$

and, therefore,

$$
\zeta_{\mathrm{QM}}=1-\frac{c_{I}}{\sqrt{c_{S} c_{L}}}=1-\frac{\left|\rho_{12}(0)\right|}{\sqrt{\rho_{11}(0) \rho_{22}(0)}}=0 \text {, }
$$

where we have used the fact that a pure quantum-mechanical $(2 \times 2)$ density matrix has zero determinant $\left[\operatorname{det} \rho(0)=\rho_{11}(0) \rho_{22}(0)-\left|\rho_{12}(0)\right|^{2}\right]$. This result applies immediately to the regenerator case where a particular form of $\rho(0) \quad$ is used: namely, $\quad \rho_{11}^{r}(0) \approx 1, \quad \rho_{22}^{r}(0) \approx|\epsilon|^{2}+$ $2 \Delta T|\epsilon| \sin \phi$, and $\left|\rho_{12}^{r}(0)\right|^{2} \approx|\epsilon|^{2}+2 \Delta T|\epsilon| \sin \phi$, which indeed satisfy $\operatorname{det} \rho^{r}(0)=0$.
We now repeat the exercise in our quantum-mechanicsviolating framework, where we obtain

$$
c_{S}^{(0+1)}=\rho_{22}(0)-\rho_{11}(0) \hat{\gamma}-2|\epsilon|\left|\rho_{12}(0)\right| \frac{\cos \left(\phi+\delta \phi+\phi_{12}\right)}{\cos \delta \phi}
$$

$$
\begin{gathered}
c_{L}^{(1+2)}=\rho_{11}(0) \hat{\gamma}+\rho_{22}(0) \hat{\gamma}^{2}+\rho_{11}(0)|\epsilon|^{2} \frac{\cos (\phi-2 \delta \phi)}{\cos \phi \cos ^{2} \delta \phi} \\
-2|\epsilon| \hat{\gamma}\left|\rho_{12}(0)\right| \frac{\cos \left(\phi-\delta \phi-\phi_{12}\right)}{\cos \delta \phi} \\
c_{I}^{(1)}=\frac{|\epsilon|}{\cos \delta \phi}\left|\rho_{12}(0)\right|
\end{gathered}
$$

which entail

$$
\begin{aligned}
\frac{c_{I}^{2}}{c_{S} c_{L}}= & \frac{|\epsilon|^{2}}{\cos ^{2} \delta \phi}\left|\rho_{12}(0)\right|^{2}\left\{\rho_{11}(0) \rho_{22}(0) \hat{\gamma}+\left[\rho_{22}^{2}(0)-\rho_{11}^{2}(0)\right] \hat{\gamma}^{2}+\rho_{11}(0) \rho_{22}(0)|\epsilon|^{2} \frac{\cos (\phi-2 \delta \phi)}{\cos \phi \cos ^{2} \delta \phi}\right. \\
& \left.-\frac{2|\epsilon| \hat{\gamma}}{\cos \delta \phi}\left|\rho_{12}(0)\right|\left[\rho_{22}(0) \cos \left(\phi-\delta \phi-\phi_{12}\right)+\rho_{11}(0) \cos \left(\phi+\delta \phi+\phi_{12}\right)\right]\right\}^{-1} .
\end{aligned}
$$

This expression can be most easily interpreted in the limit of interest, $\Delta T \gg|\epsilon|$, where the initial condition matrix $\rho^{r}(0)$ reduces to

$$
\begin{gathered}
\rho_{11}^{r}(0) \approx 1, \\
\rho_{22}^{r}(0) \approx \hat{\gamma}+2 \Delta T|\epsilon| \frac{\sin (\phi-\delta \phi)}{\cos \delta \phi}+|\epsilon|^{2} \frac{\cos (\phi-2 \delta \phi)}{\cos \phi \cos ^{2} \delta \phi},
\end{gathered}
$$

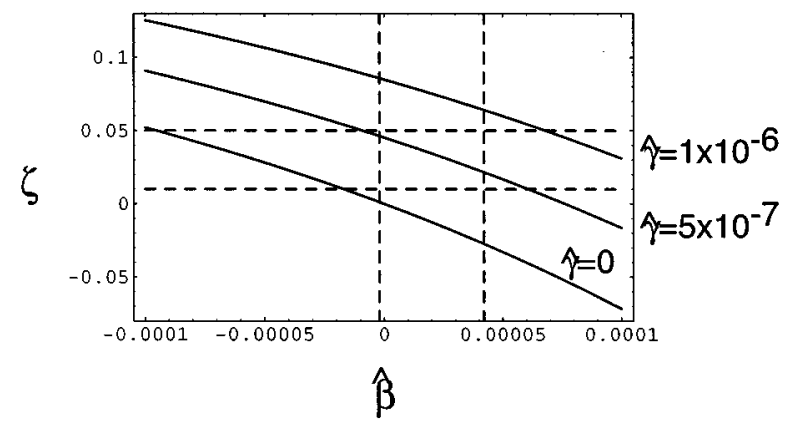

FIG. 6. The dependence of the quantum-mechanical-violating parameter $\zeta$ on $\hat{\beta}$ for representative values of $\hat{\gamma}$ ( $\hat{\alpha}$ does not contribute to the order calculated). The present experimental value of $\zeta=0.03 \pm 0.02$ is indicated, as well as our derived indicative bounds on $\hat{\beta}=(2.0 \pm 2.2) \times 10^{-5}$.

$$
\left|\rho_{12}^{r}(0)\right|^{2} \approx 2 \Delta T|\epsilon| \frac{\sin (\phi-\delta \phi)}{\cos \delta \phi}+\frac{|\epsilon|^{2}}{\cos ^{2} \delta \phi} .
$$

Note that the source of quantum-mechanical decoherence is given by

$$
\operatorname{det} \rho^{r}(0) \approx \hat{\gamma}-2|\epsilon|^{2} \frac{\sin (\phi-\delta \phi) \sin \delta \phi}{\cos \phi \cos ^{2} \delta \phi} \approx \hat{\gamma}
$$

With these expressions for $\rho^{r}(0)$, one obtains, for the numerator and denominator of Eq. (143),

$$
\begin{gathered}
c_{I}^{2} \approx \frac{|\epsilon|^{2}}{\cos ^{2} \delta \phi}\left[2 \Delta T|\epsilon| \frac{\sin (\phi-\delta \phi)}{\cos \delta \phi}\right], \\
c_{S} c_{L} \approx 2 \Delta T|\epsilon| \frac{\sin (\phi-\delta \phi)}{\cos \delta \phi}\left[\hat{\gamma}+|\epsilon|^{2} \frac{\cos (\phi-2 \delta \phi)}{\cos \phi \cos ^{2} \delta \phi}\right],
\end{gathered}
$$

and thus the regenerator effects $(\Delta T)$ drop out, and the expressions without a regenerator in Eqs. (128) and (129) are recovered, i.e., $\zeta_{r}=\zeta$. This result also implies that the experimental limits on $\zeta$, that are derived in the presence of a regenerator, can be directly applied to our expression for $\zeta$, as assumed in the previous subsection. 
We note that, although the study of $\zeta$ alone, in tests using a regenerator [24], does not seem to add anything to the discussion of the possible breakdown of quantummechanical coherence within our framework, individual terms in the expression (130) for $R_{2 \pi}^{r}(\tau)$ depend linearly on the regenerator density via $\Delta T$, and the dependence on the nonquantum-mechanical parameters is different from the noregenerator case, so the regenerator is able to provide interesting new probes of our framework. In this respect, experimental tests of $C P T$ symmetry within quantum mechanics suggested earlier [26], using arrays of regenerators, find also a natural application within our quantum-mechanicsviolating framework.
4. $A_{C P T}^{r}$

In Sec. IV D, we showed that there is no contribution to the $A_{C P T}$ observable up to second order. One may wonder whether the introduction of a regenerator could change this result. To this end we compute $A_{C P T}^{r}$, which is defined as in Eq. (78) but with the $\Delta \rho, \Sigma \rho$ matrices replaced by the $\Delta \rho^{r}, \Sigma \rho^{r}$ matrices. Expressions for the latter are complicated, as exhibited explicitly in the previous subsections. However, the expression for $A_{C P T}^{r}$ simplifies considerably when calculated consistently through first order only, since many of the entries in the input matrices $\Delta \rho\left(t_{r}\right), \Sigma \rho\left(t_{r}\right)$ need to be evaluated only to zeroth order. After some algebra, we obtain

$$
A_{C P T}^{r}(\tau)=2 \Delta T \frac{\left[e^{-\Gamma t_{r}} \sin \left(\Delta m t_{r}\right)\right]\left(e^{-\Gamma_{L} \tau}-e^{-\Gamma_{S} \tau}\right)+\left[e^{-\Gamma_{L} t_{r}}-e^{-\Gamma_{S} t_{r}}\right] e^{-\Gamma \tau_{1}} \sin \Delta(m \tau)}{e^{-\Gamma_{L^{t}}} e^{-\Gamma_{L} \tau}+e^{-\Gamma_{S} t_{r}} e^{-\Gamma_{S} \tau}+2 e^{-\Gamma t_{r}} e^{-\Gamma \tau^{\tau}} \cos \left(\Delta m \tau+\Delta m t_{r}\right)}
$$

which, for $\tau \gg \tau_{S}$, asymptotes to

$$
A_{C P T}^{r}(\tau) \rightarrow 2 \Delta T \exp \left[-\frac{1}{2}\left(\Gamma_{S}-\Gamma_{L}\right) t_{r}\right] \sin \left(\Delta m t_{r}\right) .
$$

Thus, we see that all dependence on the $C P-(|\epsilon|)$ and $C P T-(\alpha, \beta, \gamma)$ violating parameters drops out, which confirms the result obtained without a regenerator. The novelty is that $A_{C P T}^{r}$ is nonetheless nonzero, and proportional to $\Delta T$. This result is interesting, but not unexpected since the matter in the regenerator scatters $K^{0}$ differently from scattering $\bar{K}^{0}(90)$. Formally, this is expressed by the fact that the regenerator Hamiltonian in Eq. (91) is proportional to $\sigma_{1}$, and therefore does not commute with the $C P T$ operator, and so violates $C P T$. That is, the regenerator is a $C P T$-violating environment, although completely within standard quantum mechanics.

\section{INDICATIVE BOUNDS ON CPT-VIOLATING PARAMETERS}

The formulas derived above are ready to be used in fits to the experimental data. A complete analysis requires a detailed understanding of all the statistical and systematic errors, and their correlations, which goes beyond the scope of this paper [27]. Here, we restrict ourselves to indications of the magnitudes of the bounds that are likely to be obtained from such an analysis.

The parameter $\hat{\alpha}$ can be constrained by observing that the overall size of the interference term in $A_{2 \pi}$ (53) does not differ significantly from the standard result [see also Fig. 1(a)]. The relevant dependence on $\hat{\alpha}$ comes at second order through $\Delta X_{3}$, which is given in Eq. (56). From this expression, we can see that the dominant term is the third one, i.e., $(-2|\epsilon| \hat{\alpha} / \cos \delta \phi) t|\Delta \Gamma| \cos (\Delta m t-\phi-\delta \phi)$, which is enhanced relative to the other terms because of the $t|\Delta \Gamma|$ factor. The dominant interference term through second order is then

$$
-\frac{2|\epsilon|}{\cos \delta \phi}[1+\hat{\alpha} t|\Delta \Gamma|] e^{\left(\Gamma_{S}-\Gamma_{L}\right) t / 2} \cos (\Delta m t-\phi-\delta \phi) .
$$

For our indicative purposes, we assume that the size of the interference term is within $5 \%$ of the standard result for observations in the range $t / \tau_{S} \leqslant 10$. Since $\hat{\alpha}>0$ and the overall factor $(1 / \cos \delta \phi) \approx 1$ (see below), we require $\hat{\alpha} t|\Delta \Gamma| \lesssim 0.05$ [16]: i.e.,

$$
\hat{\alpha} \lesssim 5.0 \times 10^{-3}, \quad \alpha \lesssim 3.7 \times 10^{-17} \mathrm{GeV} .
$$

This is to be compared to the order of magnitude $O\left(\left(\Lambda_{\mathrm{QCD}} \text { or } m_{s}\right)^{2} / M_{\mathrm{PI}}\right) \leq 10^{-19} \mathrm{GeV}$ which is of theoretical interest in the neutral kaon system.

The simplest way to constrain the parameter $\hat{\beta}$ involves the observables $R_{2 \pi}$ and $A_{2 \pi}$, which differ from the standard results at first order in $\hat{\beta}$, as seen in Fig. 1(b). This new contribution can affect the overall size of the interference pattern and shift its phase relative to the superweak phase $\phi$, as seen in Eqs. (53) and (133). It is easy to check that the shift in phase $\delta \phi$ is sufficiently small for any possible change in the overall size of the interference pattern (because of $\delta \phi$ ) to be negligible, e.g., $|\delta \phi|<2^{\circ}$ implies a change in the size by $<6 \times 10^{-4}$. There are two independent sets of data that give information on $\delta \phi$ : (i) the Particle Data Group compilation [1] which fits NA31, E731, and earlier data, and (ii) more recent data from the E773 Collaboration [22,23]. New data from the CPLEAR Collaboration are discussed elsewhere [27]. In each case, both the superweak phase $\phi$ and the $K \rightarrow \pi^{+} \pi^{-}$interference phase $\phi_{+-}$are measured, and the corresponding values of $\delta \phi=\phi_{+_{-}-} \phi$ are extracted:

$$
\delta \phi=(-0.71 \pm 0.95)^{\circ}[1], \quad \delta \phi=(-0.84 \pm 1.42)^{\circ} \quad[22] .
$$


Combining these independent measurements in quadrature, we find $\delta \phi=(-0.75 \pm 0.79)^{\circ}$, corresponding to

$$
\hat{\beta}=(2.0 \pm 2.2) \times 10^{-5}, \quad \beta=(1.5 \pm 1.6) \times 10^{-19} \mathrm{GeV},
$$

to be compared with the earlier bound $|\hat{\beta}| \lesssim 6 \times 10^{-5}$ obtained in Ref. [16] by demanding $|\delta \phi| \lesssim 2^{\circ}$. As expected from Fig. 1, the indicative bound (155) on $|\hat{\beta}|$ is considerably more restrictive than that (153) on $|\hat{\alpha}|$. Alternatively, one may bound $\hat{\beta}$ by considering the relationship (see, e.g., [23])

$$
\left|m_{K^{0}}-m_{K^{0}}\right| \approx 2 \Delta m \frac{\left|\eta_{+-}\right|}{\sin \Phi_{\mathrm{sw}}}\left|\Phi_{+-}-\Phi_{\mathrm{sw}}+\frac{1}{3} \Delta \Phi\right|,
$$

where $\Delta \Phi=\Phi_{00}-\Phi_{+-}$. As pointed out in Ref. [19], Eq. (156) is modified in the presence of direct $C P T$ violation in the $I=0 \quad K_{L} \rightarrow 2 \pi$ decay amplitude [see Eq. (13) of Ref. [19]]. Consequences of such direct $C P T$ violation are addressed in the concluding section of this paper [see our Eqs. (169)-(174)].

In our particular framework, up to $\epsilon^{\prime} / \epsilon$ effects, $\Delta \Phi=0$, $\Phi_{\mathrm{sw}}=\phi, \Phi_{+-}=\phi+\delta \phi,\left|\eta_{+-}\right|=|\epsilon| / \cos \delta \phi \approx|\epsilon|$, and thus

$$
\left|m_{K^{0}}-m_{K^{0}}\right| \approx 2 \Delta m \frac{|\epsilon||\delta \phi|}{\sin \phi} \approx 2|\beta| .
$$

The E773 Collaboration has determined [23] that $\left|m_{K^{0}}-m_{K^{0}}\right| / m_{K^{0}}<13 \times 10^{-19}$ at the $90 \%$ C.L., whereas the Particle Data Group quotes $\left|m_{K^{0}}-m_{K^{0}}\right| / m_{K^{0}}<9 \times 10^{-19}[1]$, and thus it follows that $|\hat{\beta}|<3.1 \times 10^{-5}$, $|\beta|<2.3 \times 10^{-19} \mathrm{GeV}$. This result is consistent with that in Eq. (155).

The $\hat{\gamma}$ parameter has the peculiar property of appearing in the observables at first order, but without being accompanied by a similar first-order term proportional to $|\epsilon|$ (as is the case for $\hat{\beta})$. In fact, if corresponding terms exist, they are proportional to $|\epsilon|^{2}$. This means that large deviations from the usual results would occur unless $\hat{\gamma} \lesssim|\epsilon|^{2}$. This result is exemplified in Fig. 1(c), from which we conclude that $\hat{\gamma}<10^{-5}$. In Ref. [16], $\hat{\gamma} \lesssim 0.1|\epsilon|^{2}$ was obtained. However, since $\left|\epsilon^{\prime} / \epsilon\right| \sim 10^{-3}$ effects have been neglected, we conclude conservatively that

$$
\hat{\gamma} \lesssim\left|\frac{\epsilon^{\prime}}{\epsilon}\right||\epsilon| \sim 10^{-6}, \quad \gamma \lesssim 7 \times 10^{-21} \mathrm{GeV} .
$$

We can also study the combined effects of $\hat{\beta}$ and $\hat{\gamma}$ on the $\zeta$ parameter in Eq. (129), which reads

$$
\frac{\hat{\gamma}}{2|\epsilon|^{2}}-\frac{2 \hat{\beta}}{|\epsilon|} \sin \phi=0.03 \pm 0.02
$$

The combined bounds on both parameters can be read off Fig. 6, which makes clearly the point that a combined fit is essential to obtain the true bounds on the $C P T$-violating parameters. Note that the bounds on $\hat{\beta}$ (155) and $\hat{\gamma}$ (158) derived above are consistent with those that follow from Eq. (159) (see Fig. 6).
TABLE I. Compilation of indicative bounds on $C P T$-violating parameters and their sources.

\begin{tabular}{lc}
\hline \hline Source & Indicative bound \\
\hline$R_{2 \pi}, A_{2 \pi}$ & $\hat{\alpha}<5.0 \times 10^{-3}$ \\
$R_{2 \pi}, A_{2 \pi}$ & $\hat{\beta}=(2.0 \pm 2.2) \times 10^{-5}$ \\
$\left|m_{K^{0}}-m_{K^{0}}\right|$ & $\hat{\beta}<3.1 \times 10^{-5}$ \\
$R_{2 \pi}$ & $\hat{\gamma} \lesssim 5 \times 10^{-7}$ \\
$\zeta$ & $\frac{\hat{\gamma}}{2|\epsilon|^{2}}-\frac{2 \hat{\beta}}{|\epsilon|} \sin \phi=0.03 \pm 0.02$ \\
Positivity & $\hat{\alpha}>\hat{\beta}^{2} / \hat{\gamma}_{\max } \sim\left(10^{3} \hat{\beta}\right)^{2}$ \\
\hline \hline
\end{tabular}

Let us close this section with a remark concerning the positivity constraints in Eq. (14): $\alpha>0, \gamma>0$, and $\alpha \gamma>\beta^{2}$. The data are not yet sufficient to conclude anything about the sign of the $\alpha$ and $\gamma$ parameters. The third constraint implies

$$
\hat{\alpha}>\frac{\hat{\beta}^{2}}{\hat{\gamma}}>\frac{\hat{\beta}^{2}}{\hat{\gamma}_{\max }} \sim\left(10^{3} \hat{\beta}\right)^{2} .
$$

Thus, if $\beta$ is observable, say $\hat{\beta} \sim 10^{-5}$, then $\hat{\alpha}>10^{-4}$ should be observable too. A compilation of all these indicative bounds and their sources is given in Table I.

\section{COMMENT ON TWO-PARTICLE DECAY CORRELATIONS}

Further interesting tests of quantum mechanics and $C P T$ symmetry can be devised by exploiting initial-state correlations because of the production of a pair of neutral kaons in a pure quantum-mechanical state, e.g., via $e^{+} e^{-} \rightarrow \phi \rightarrow$ $K^{0} \bar{K}^{0}$. In this case, the initial state may be represented by [28]

$$
|\mathbf{k} ;-\mathbf{k}\rangle=\frac{1}{\sqrt{2}}\left[\left|K^{0}(\mathbf{k}) ; \bar{K}^{0}(-\mathbf{k})\right\rangle-\left|\bar{K}^{0}(\mathbf{k}) ; K^{0}(-\mathbf{k})\right\rangle\right] .
$$

At subsequent times $t=t_{1}$ for particle 1 and $t=t_{2}$ for particle 2 , the joint probability amplitude is given in conventional quantum mechanics by

$$
\left|\mathbf{k}, t_{1} ;-\mathbf{k}, t_{2}\right\rangle \equiv e^{-i H(\mathbf{k}) t_{1}} e^{-i H(-\mathbf{k}) t_{2}}|\mathbf{k} ;-\mathbf{k}\rangle .
$$

Thus, the temporal evolution of the two-particle state is completely determined by the one-particle variables (OPV's) contained in $H$.

Tests of quantum mechanics and CPT symmetry in $\phi$ decays have recently been discussed [17] in a conjectured extension of the formalism of $[6,15]$, in which the density matrix of the two-particle system was hypothesized to be described completely in terms of such one-particle variables (OPV's): namely, $H$ and $(\alpha, \beta, \gamma)$. It was pointed out that this OPV hypothesis had several striking consequences, including apparent violations of energy conservation and angular momentum. 
As we have discussed above [29], the only known theoretical framework in which Eq. (2) has been derived is that of a noncritical string approach to string theory, in which (i) energy is conserved in the mean as a consequence of the renormalizability of the world-sheet $\sigma$ model, but (ii) angular momentum is not necessarily conserved [15,9], as this is not guaranteed by renormalizability and is known to be violated in some toy backgrounds [29], though we cannot exclude the possibility that it may be conserved in some particular background(s). Therefore, we are not concerned that Huet and Peskin [17] find angular momentum nonconservation in their hypothesized OPV approach. However, the absence of energy conservation in their approach leads us to the conclusion that irreducible two-particle parameters must be introduced into the evolution of the two-particle density matrix. The appearance of such nonlocal parameters does not concern us, as the string is intrinsically nonlocal in target space, and this fact plays a key role in our model calculations of contributions to $\delta H$. The justification and parametrization of such irreducible two-particle effects go beyond the scope of this paper, and we plan to study this subject in more detail in due course.

\section{CONCLUSIONS}

We have derived in this paper approximate expressions for a complete set of neutral kaon decay observables $(2 \pi, 3 \pi, \pi \ell \nu)$ which can be used to constrain parameters characterizing $C P T$ violation in a formalism, motivated by ideas about quantum gravity and string theory, that incorporates a possible microscopic loss of quantum coherence by treating the neutral kaon as an open quantum-mechanical system. Our explicit expressions are to second order in the small $C P T$-violating parameters $\alpha, \beta, \gamma$, and our systematic procedure for constructing analytic approximations may be extended to any desired level of accuracy. Our formulas may be used to obtain indicative upper bounds

$$
\begin{gathered}
\alpha \lesssim 4 \times 10^{-17} \mathrm{GeV}, \quad|\beta| \lesssim 3 \times 10^{-19} \mathrm{GeV}, \\
\gamma \lesssim 7 \times 10^{-21} \mathrm{GeV},
\end{gathered}
$$

which are comparable with the order of magnitude $\sim 10^{-19} \mathrm{GeV}$ which theory indicates might be attained by such $C P T$ - and quantum-mechanics-violating parameters. Detailed fits to recent CPLEAR experimental data are reported elsewhere [27].

We have not presented explicit expressions for the case where the deviation $\left|\epsilon^{\prime} / \epsilon\right| \lesssim 10^{-3}$ from pure superweak $C P$ violation is non-negligible, but our methods can easily be extended to this case. They can also be used to obtain more specific expressions for experiments with a regenerator, if desired. The extension of the formalism of Ref. [6] to correlated $K^{0} \bar{K}^{0}$ systems produced in $\phi$ decay, as at DA $\Phi$ NE [4], involves the introduction of two-particle variables, which lies beyond the scope of this paper.

As mentioned in the main text, in Appendix A we have obtained formulas for all observables in the case of $C P T$ violation within standard quantum mechanics. In the case of $A_{2 \pi}^{\mathrm{QM}}$ and $A_{3 \pi}^{\mathrm{QM}}$, one can "mimic" the results from standard $C P$ violation with suitable choices of the $C P T$-violating pa-
TABLE II. Qualitative comparison of predictions for various observables in $C P T$-violating theories beyond quantum-mechanics violation $(\mathrm{QMV})$ and within quantum mechanics $(\mathrm{QM})$. Predictions either differ $(\neq)$ or agree $(=)$ with the results obtained in conventional quantum-mechanical $C P$ violation. Note that these frameworks can be qualitatively distinguished via their predictions for $A_{\mathrm{T}}, A_{\mathrm{CPT}}, A_{\Delta m}$, and $\zeta$.

\begin{tabular}{lcl}
\hline \hline Process & QMV & QM \\
\hline$A_{2 \pi}$ & $\neq$ & $\neq$ \\
$A_{3 \pi}$ & $\neq$ & $\neq$ \\
$A_{\mathrm{T}}$ & $\neq$ & $=$ \\
$A_{C P T}$ & $=$ & $\neq$ \\
$A_{\Delta m}$ & $\neq$ & $=$ \\
$\zeta$ & $\neq$ & $=$ \\
\hline \hline
\end{tabular}

rameters $(\delta M=0,>\widehat{\delta \Gamma} \rightarrow-2|\epsilon| / \cos \phi)$. However, this possibility is experimentally excluded because of the large value it entails for the $A_{C P T}$ observable. In passing, we showed that the $\zeta$ parameter vanishes since no violation of quantum mechanics is allowed. In analogy with Sec. VI, we also obtained indicative bounds on the $C P T$-violating parameters. In Table II we list all the observables and make a qualitative comparison between them and conventional quantum-mechanical $C P$ violation. We see that the $\mathrm{QM}$ and quantum-mechanicsviolating (QMV) $C P T$-violating frameworks can be qualitatively distinguished by their predictions for $A_{\mathrm{T}}, A_{C P T}$, $A_{\Delta m}$, and $\zeta$. As seen in Table II, the two observables $A_{2 \pi}$ and $A_{3 \pi}$ are sensitive to CPT violation in both the QM and QMV frameworks. For the sake of completeness, we present here formulas that include both these possible effects, and discuss how they may be disentangled.

Given the present status of experimental activities, the more sensitive observable is $A_{2 \pi}$ in the interference region, where first-order effects are dominant. In this case, we obtain an expression for $A_{2 \pi}$ which resembles Eq. (53) (with $\left.\Sigma X_{1,2,3}=\Delta X_{1,2,3}=0\right)$, but with the replacements

$$
\delta \phi \rightarrow \delta \phi_{X}, \quad|\epsilon| \rightarrow|\epsilon|\left[1-\frac{1}{2} \frac{\widehat{\delta \Gamma}}{|\epsilon|} \cos \phi\right] \approx|\epsilon|
$$

where the new phase shift $\delta \phi_{X}$ is defined by

$$
\tan \delta \phi_{X}=-\frac{(2 \hat{\beta}-\widehat{\delta M}) \cos \phi}{|\epsilon|\left[1-\frac{1}{2} \frac{\widehat{\delta \Gamma}}{|\epsilon|} \cos \phi\right]} \approx-\frac{(2 \hat{\beta}-\widehat{\delta M})}{|\epsilon|} \cos \phi
$$

This generalized expression implies that the indicative bounds in Eqs. (155) and (A43) become combined into

$$
\hat{\beta}-\frac{1}{2} \widehat{\delta M}=(2.0 \pm 2.2) \times 10^{-5}
$$


This implies that the effects of $\hat{\beta}$ and $\delta M$ cannot be disentangled by a measurement of $A_{2 \pi}$ alone. Recall, however, that these parameters appear in different conceptual frameworks, so there is no strong reason to expect both them of to be present simultaneously. Turning to the $A_{3 \pi}$ observable, combining the effects of both QM and QMV sources of $C P T$ violation leads to the replacements in Eq. (68):

$$
\delta \phi \rightarrow \delta \phi_{X^{\prime}}, \quad|\epsilon| \rightarrow|\epsilon|\left[1+\frac{1}{2} \frac{\widehat{\delta \Gamma}}{|\epsilon|} \cos \phi\right] \approx|\epsilon|,
$$

where the new phase shift $\delta \phi_{X^{\prime}}$ is defined by

$$
\tan \delta \phi_{X^{\prime}}=-\frac{(2 \hat{\beta}-\widehat{\delta M}) \cos \phi}{|\epsilon|\left[1+\frac{1}{2} \frac{\delta \Gamma}{|\epsilon|} \cos \phi\right]} \approx \tan \delta \phi_{X} .
$$

We note that a non-negligible value of $\delta \Gamma$ would be indicated if the phase shifts in $A_{2 \pi}$ and $A_{3 \pi}$ should be found to differ, i.e., $\delta \phi_{X}$ vs $\delta \phi_{X^{\prime}} \cdot{ }^{5}$ Any such possible discrepancy would not, however, reveal whether QMV CPT violation (i.e., $\hat{\beta}$ ) is present or not.

We comment also on the possible appearance of direct $C P T$ violation in decay amplitudes. Such phenomena are not predicted by the modification to quantum mechanics that we have discussed above, but have been proposed elsewhere [19]. If such an effect were present, the two-pion decay operator in Eq. (17) would be generalized to

$$
O_{2 \pi}^{\prime}=\left(\begin{array}{cc}
|\lambda|^{2} & \lambda \\
\lambda^{*} & 1
\end{array}\right)
$$

where $\lambda$ is a new complex $C P T$-violating parameter. The first-order result for the $A_{2 \pi}$ observable is then shifted by

$$
-2|\lambda| e^{\left(\Gamma_{S}-\Gamma_{L}\right) t / 2} \cos \left(\Delta m t-\phi_{\lambda}\right),
$$

where we have defined $\lambda=|\lambda| e^{i \phi_{\lambda}}$. Since the amplitude of the cosine term in $K \rightarrow 2 \pi$ is known not to differ much from the usual result, we see that $|\lambda| /|\epsilon| \ll 1$ is required. We can combine this new contribution to $A_{2 \pi}$ with the previous ones to generalize the previous results for $\left|\eta_{+-}\right|=|\epsilon| / \cos \delta \phi \approx|\epsilon|$ and $\phi_{+-}=\phi+\delta \phi$. To first order in $|\lambda| /|\epsilon|$, we find

$$
\begin{gathered}
\left|\eta_{+-}\right| \approx|\epsilon|+|\lambda| \cos \left(\phi-\phi_{\lambda}\right), \\
\phi_{+-} \approx \phi+\delta \phi-\frac{|\lambda|}{|\epsilon|} \sin \left(\phi-\phi_{\lambda}\right) .
\end{gathered}
$$

(Note that $\lambda$ causes a first-order shift in $\left|\eta_{+-}\right|$, whereas $\beta$ first shifts it at second order.) The expression for $\left|m_{K^{0}}-m_{\bar{K}^{0}}\right|$ in Eq. (156) is generalized to

$$
\left|m_{K^{0}}-m_{\bar{K}^{0}}\right| \approx|2 \beta+| \lambda|| \Delta \Gamma\left|\frac{\sin \left(\phi-\phi_{\lambda}\right)}{\cos \phi}\right| .
$$

\footnotetext{
${ }^{5}$ This possibility is not allowed in the $C P T$-violating scenario of Ref. [18], where $\delta \Gamma=0$ is obtained.
}

If there were direct $C P T$ violation in the $\pi \ell \nu$ decay amplitudes, the semileptonic observables (18) would also be modified, becoming

$$
O_{\pi^{-} l^{+} \nu}^{\prime}=|A|^{2} O_{\pi^{-} l^{+} \nu}, \quad O_{\pi^{+} l^{-} \bar{\nu}}=|\bar{A}|^{2} O_{\pi^{+} l^{-} \bar{\nu}},
$$

where any difference between $A$ and $\bar{A}$ would violate $C P T$. Since $C P T$ violation must be small, we expect $|A| /|\bar{A}| \equiv 1+\eta \approx 1$ and we can then examine the effects of $\eta$ to first order. These effects enter in $A_{\mathrm{T}}, A_{\Delta m}$, and $A_{C P T}$. However, they are most relevant in the last observable, as the prediction for it in QMV vanishes. A calculation along the lines of that in Sec. IV D gives to first order $A_{C P T}^{\prime}=2 \eta$, offering a clear distinction from the QMV scenario. We recall that the CPLEAR Collaboration has reported the result $A_{C P T}^{\text {expt }}=(-0.4 \pm 2.0 \pm 2.0 \pm 1.5) \times 10^{-3}$ [3], confirming the smallness of $\eta$.

We close by reiterating that the neutral kaon system is the best microscopic laboratory for testing quantum mechanics and $C P T$ symmetry. We believe that violations of these two fundamental principles, if present at all, are likely to be linked, and have proposed a formalism that can be used to explore systematically this hypothesis, which is motivated by ideas about quantum gravity and string theory. Our understanding of these difficult issues is so incomplete that we cannot calculate the sensitivity which would be required to reveal modifications of quantum mechanics or a violation of $C P T$. Hence, we cannot promise success in any experimental search for such phenomena. However, we believe that both the theoretical and experimental communities should be open to their possible appearances.

\section{ACKNOWLEDGMENTS}

We would like to thank P. Eberhard, P. Huet, P. Pavlopoulos, and T. Ruf for useful discussions. The work of N.E.M. was supported by the European Union Research Foundation Fellowship, Proposal No. ERB4001GT922259, and that of D.V.N. in part by DOE Grant No. DE-FG05-91-ER-40633. J.E. thanks P. Sorba and the LAPP Laboratory for hospitality during work on this subject. N.E.M. also thanks D. Cocolicchio, G. Pancheri, N. Paver, and other members of the DAФNE working groups for their interest in this work.

\section{APPENDIX A: $C P T$ VIOLATION IN THE QUANTUM-MECHANICAL DENSITY MATRIX FORMALISM FOR NEUTRAL KAONS}

In this appendix we review the density-matrix formalism for neutral kaons and $C P T$ violation within the conventional quantum-mechanical framework $[5,15]$. The time evolution of a generic density matrix is determined in this case by the usual quantum Liouville equation

$$
\partial_{t} \rho=-i\left(H \rho-\rho H^{\dagger}\right) .
$$

The conventional phenomenological Hamiltonian for the neutral kaon system contains Hermitian (mass) and antiHermitian (decay) components: 


$$
H=\left(\begin{array}{cc}
\left(M+\frac{1}{2} \delta M\right)-\frac{1}{2} i\left(\Gamma+\frac{1}{2} \delta \Gamma\right) & M_{12}^{*}-\frac{1}{2} i \Gamma_{12}^{*} \\
M_{12}-\frac{1}{2} i \Gamma_{12} & \left(M-\frac{1}{2} \delta M\right)-\frac{1}{2} i\left(\Gamma-\frac{1}{2} \delta \Gamma\right)
\end{array}\right),
$$

in the $\left(K^{0}, \bar{K}^{0}\right)$ basis. The $\delta M$ and $\delta \Gamma$ terms violate $C P T$ [5]. As in Ref. [6], we define components of $\rho$ and $H$ by

$$
\rho \equiv \frac{1}{2} \rho_{\alpha} \sigma_{\alpha}, \quad H \equiv \frac{1}{2} h_{\alpha} \sigma_{\alpha}, \quad \alpha=0,1,2,3
$$

in a Pauli $\sigma$-matrix representation: the $\rho_{\alpha}$ are real, but the $h_{\beta}$ are complex. The $C P T$ transformation is represented by

$$
C P T\left|K^{0}\right\rangle=e^{i \theta}\left|\bar{K}^{0}\right\rangle, \quad C P T\left|\bar{K}^{0}\right\rangle=e^{-i \theta}\left|K^{0}\right\rangle,
$$

for some phase $\theta$, which is represented in our matrix formalism by

$$
C P T \equiv\left(\begin{array}{ll}
0 & e^{i \theta} \\
e^{-i \theta} & 0
\end{array}\right)
$$

Since this matrix is a linear combination of $\sigma_{1,2}, C P T$ invariance of the phenomenological Hamiltonian, $H=$ $(C P T)^{-1} H(C P T)$, clearly requires that $H$ contains no term proportional to $\sigma_{3}$, i.e., $h_{3}=0$ so that $\delta M=\delta \Gamma=0$.

Conventional quantum-mechanical evolution is represented by $\partial_{t} \rho_{\alpha}=H_{\alpha \beta} \rho_{\beta}$, where, in the $\left(K^{0}, \bar{K}^{0}\right)$ basis and allowing for the possibility of $C P T$ violation,

$$
H_{\alpha \beta} \equiv\left(\begin{array}{cccc}
\operatorname{Im} h_{0} & \operatorname{Im} h_{1} & \operatorname{Im} h_{2} & \operatorname{Im} h_{3} \\
\operatorname{Im} h_{1} & \operatorname{Im} h_{0} & -\operatorname{Re} h_{3} & \operatorname{Re} h_{2} \\
\operatorname{Im} h_{2} & \operatorname{Re} h_{3} & \operatorname{Im} h_{0} & -\operatorname{Re} h_{1} \\
\operatorname{Im} h_{3} & -\operatorname{Re} h_{2} & \operatorname{Re} h_{1} & \operatorname{Im} h_{0}
\end{array}\right) .
$$

We note that the real parts of the matrix $h$ are antisymmetric, while its imaginary parts are symmetric. Now is an appropriate time to transform to the $K_{1,2}=1 / \sqrt{2}\left(K^{0} \mp \bar{K}^{0}\right)$ basis, corresponding to $\sigma_{1} \leftrightarrow \sigma_{3}, \sigma_{2} \leftrightarrow-\sigma_{2}$, in which $H_{\alpha \beta}$ becomes

$$
H_{\alpha \beta}=\left(\begin{array}{cccc}
-\Gamma & -\frac{1}{2} \delta \Gamma & -\operatorname{Im} \Gamma_{12} & -\operatorname{Re} \Gamma_{12} \\
-\frac{1}{2} \delta \Gamma & -\Gamma & -2 \operatorname{Re} M_{12} & -2 \operatorname{Im} M_{12} \\
-\operatorname{Im} \Gamma_{12} & 2 \operatorname{Re} M_{12} & -\Gamma & -\delta M \\
-\operatorname{Re} \Gamma_{12} & -2 \operatorname{Im} M_{12} & \delta M & -\Gamma
\end{array}\right) .
$$

The corresponding equations of motion for the components of $\rho$ in the $K_{1,2}$ basis are (as above we neglect $\operatorname{Im} \Gamma_{12}$ contributions)

$$
\dot{\rho}_{11}=-\Gamma_{L} \rho_{11}-2 \operatorname{Re}\left[\left(\operatorname{Im} M_{12}+\frac{1}{4} \delta \Gamma+\frac{i}{2} \delta M\right) \rho_{12}\right],
$$

$$
\begin{aligned}
\dot{\rho}_{12}= & -(\Gamma+i \Delta m) \rho_{12}+\left(\operatorname{Im} M_{12}-\frac{1}{4} \delta \Gamma-\frac{i}{2} \delta M\right) \rho_{11} \\
& -\left(\operatorname{Im} M_{12}+\frac{1}{4} \delta \Gamma-\frac{i}{2} \delta M\right) \rho_{22}, \\
\dot{\rho}_{22}= & -\Gamma_{S} \rho_{22}+2 \operatorname{Re}\left[\left(\operatorname{Im} M_{12}-\frac{1}{4} \delta \Gamma+\frac{i}{2} \delta M\right) \rho_{12}\right] .
\end{aligned}
$$

One can readily verify that $\rho$ decays at large $t$ to

$$
\rho \sim e^{-\Gamma_{L} t}\left(\begin{array}{cc}
1 & \epsilon^{*}+\delta^{*} \\
\epsilon+\delta & |\epsilon+\delta|^{2}
\end{array}\right),
$$

which has a vanishing determinant, thus corresponding to a pure long-lived mass eigenstate $K_{L}$. The $C P$-violating parameter $\epsilon$ and the $C P T$-violating parameter $\delta$ are given as above: namely,

$$
\epsilon=\frac{\operatorname{Im} M_{12}}{\frac{1}{2}|\Delta \Gamma|+i \Delta m}, \quad \delta=-\frac{1}{2} \frac{\frac{1}{2} \delta \Gamma+i \delta M}{\frac{1}{2}|\Delta \Gamma|+i \Delta m} .
$$

Conversely, in the short- $t$ limit, a $K_{S}$ state is represented by

$$
\rho \sim e^{-\Gamma_{S^{t}}}\left(\begin{array}{cc}
|\epsilon-\delta|^{2} & \epsilon-\delta \\
\epsilon^{*}-\delta^{*} & 1
\end{array}\right),
$$

which also has zero determinant. Note that the relative signs of the $\delta$ terms have reversed: this is the signature of $C P T$ violation in the conventional quantum-mechanical formalism. Note that the density matrices [Eqs. (A11) and (A13)] correspond to the state vectors

$$
\begin{gathered}
\left|K_{L}\right\rangle \propto(1+\epsilon-\delta)\left|K^{0}\right\rangle-(1-\epsilon+\delta)\left|\bar{K}^{0}\right\rangle, \\
\left|K_{S}\right\rangle \propto(1+\epsilon+\delta)\left|K^{0}\right\rangle+(1-\epsilon-\delta)\left|\bar{K}^{0}\right\rangle,
\end{gathered}
$$

and are both pure, as should be expected in conventional quantum mechanics, even if $C P T$ is violated.

As above, we solve the differential equations in perturbation theory in $|\epsilon|$ and the new parameters

$$
\widehat{\delta M} \equiv \frac{\delta M}{|\Delta \Gamma|}, \quad \widehat{\delta \Gamma} \equiv \frac{\delta \Gamma}{|\Delta \Gamma|} .
$$

The zeroth order results for the $\rho_{i j}$ are the same as those in Eqs. (36)-(38): namely,

$$
\begin{gathered}
\rho_{11}^{(0)}(t)=\rho_{11}(0) e^{-\Gamma_{L^{t}},} \\
\rho_{22}^{(0)}(t)=\rho_{22}(0) e^{-\Gamma_{S^{t}},} \\
\rho_{12}^{(0)}(t)=\rho_{12}(0) e^{-(\Gamma+i \Delta m t) .}
\end{gathered}
$$


The first-order results for the density matrix elements are

$$
\begin{aligned}
& \rho_{11}^{(1)}=-2\left|X^{\prime}\right|\left|\rho_{12}(0)\right|\left[e^{-\Gamma_{L^{t}} \cos \left(\phi-\phi_{X^{\prime}}-\phi_{12}\right)}\right. \\
& \left.-e^{-\Gamma t} \cos \left(\Delta m t+\phi-\phi_{X^{\prime}}-\phi_{12}\right)\right], \\
& \rho_{22}^{(1)}=-2|X|\left|\rho_{12}(0)\right|\left[e^{-\Gamma_{S} t} \cos \left(\phi+\phi_{X}+\phi_{12}\right)\right. \\
& \left.-e^{-\Gamma t} \cos \left(\Delta m t-\phi-\phi_{X}-\phi_{12}\right)\right] \text {, } \\
& \rho_{12}^{(1)}=\rho_{11}(0)|X| e^{-i\left(\phi+\phi_{X}\right)}\left[e^{-\Gamma_{L} t}-e^{-(\Gamma+i \Delta m) t}\right] \\
& +\rho_{22}(0)\left|X^{\prime}\right| e^{i\left(\phi-\phi_{X^{\prime}}\right)}\left[e^{-\Gamma_{S} t}-e^{-(\Gamma+i \Delta m) t}\right],
\end{aligned}
$$

where the two complex constants $X$ and $X^{\prime}$ are defined by $X=|\epsilon|-\frac{1}{2} \cos \phi \widehat{\delta \Gamma}+i \cos \phi \widehat{\delta M}, \quad \tan \phi_{X}=\frac{\cos \phi \widehat{\delta M}}{|\epsilon|-\frac{1}{2} \cos \phi \widehat{\delta \Gamma}}$,

$$
\begin{gathered}
X^{\prime}=|\epsilon|+\frac{1}{2} \cos \phi \widehat{\delta \Gamma}+i \cos \phi \widehat{\delta M} \\
\tan \phi_{X^{\prime}}=\frac{\cos \phi \widehat{\delta M}}{|\epsilon|+\frac{1}{2} \cos \phi \widehat{\delta \Gamma}} .
\end{gathered}
$$

For future reference, we note the special case that occurs when $\delta M=0$ : and $|\epsilon|=0$, namely,

$$
\begin{array}{ccc}
\delta \Gamma>0: & \phi_{X}=\pi, & \phi_{X^{\prime}}=0 \\
\delta \Gamma<0: & \phi_{X}=0, & \phi_{X^{\prime}}=\pi .
\end{array}
$$

With the results for $\rho$ through first order, and inserting the appropriate initial conditions (28), we can immediately write down the expressions for the various observables discussed in Sec. IV. For $A_{2 \pi}$, we obtain

$$
A_{2 \pi}^{\mathrm{QM}}(t)=\frac{2|X| \cos \left(\phi+\phi_{X}\right)-2|X| e^{\left(\Gamma_{S}-\Gamma_{L}\right) t / 2} \cos \left(\Delta m t-\phi-\phi_{X}\right)}{1+e^{\left(\Gamma_{S}-\Gamma_{L}\right) t}|X|^{2}},
$$

where in the denominator we have also included the nonnegligible second-order contributions to $\Sigma \rho_{22}^{(2)}$. From this expression, it is interesting to note that one can mimic the standard $C P$-violating result for $A_{2 \pi}$ in Eq. (60) by setting $|\epsilon| \rightarrow 0$ and making the choices for the $C P T$-violating parameters

$$
\text { mimic } C P \text { violation: } \quad \delta M=0, \quad \widehat{\delta \Gamma} \rightarrow-\frac{2|\epsilon|}{\cos \phi},
$$

which give $|X| \rightarrow|\epsilon|$ and $\phi_{X}=0$. For the $A_{3 \pi}$ observable, we find

$$
\begin{aligned}
A_{3 \pi}^{\mathrm{QM}}(t)= & 2\left|X^{\prime}\right| \cos \left(\phi-\phi_{X^{\prime}}\right)-2 e^{-\left(\Gamma_{S}-\Gamma_{L}\right) t / 2}\left[\operatorname{Re} \eta_{3 \pi} \cos \Delta m t\right. \\
& \left.-\operatorname{Im} \eta_{3 \pi} \sin \Delta m t\right],
\end{aligned}
$$

with

$$
\operatorname{Re} \eta_{3 \pi}=\left|X^{\prime}\right| \cos \left(\phi-\phi_{X^{\prime}}\right), \quad \operatorname{Im} \eta_{3 \pi}=\left|X^{\prime}\right| \sin \left(\phi-\phi_{X^{\prime}}\right),
$$

that is

$$
\frac{\operatorname{Im} \eta_{3 \pi}}{\operatorname{Re} \eta_{3 \pi}}=\tan \left(\phi-\phi_{X^{\prime}}\right) .
$$

Here, we also note that the standard $C P$-violating result is obtained for the choices of parameters in Eq. (A28) which give $\left|X^{\prime}\right| \rightarrow|\epsilon|$ and $\phi_{X^{\prime}}=\pi$, since $\tan (\phi-\pi)=\tan \phi$.

For the observable $A_{\mathrm{T}}$, we obtain the exactly timeindependent first-order expression

$$
A_{\mathrm{T}}^{\mathrm{QM}}=2\left|X^{\prime}\right| \cos \left(\phi-\phi_{X^{\prime}}\right)+2|X| \cos \left(\phi+\phi_{X}\right)=4|\epsilon| \cos \phi,
$$

which is identical to the case of no- $C P T$ violation. In the case of $A_{C P T}$, we find

$$
A_{C P T}^{\mathrm{QM}}(t)=\frac{A_{1}\left(e^{-\Gamma_{L} t}-e^{-\Gamma_{S} t}\right)-2 e^{-\Gamma t} A_{2} \sin \Delta m t}{e^{-\Gamma_{L} t}+e^{-\Gamma_{S} t}-2 e^{-\Gamma t} \cos \Delta m t},
$$

with

$$
\begin{aligned}
A_{1} & =2\left|X^{\prime}\right| \cos \left(\phi-\phi_{X^{\prime}}\right)-2|X| \cos \left(\phi+\phi_{X}\right) \\
& =4 \sin \phi \cos \phi \widehat{\delta M}+2 \cos ^{2} \phi \widehat{\delta \Gamma} \\
A_{2} & =-2\left|X^{\prime}\right| \sin \left(\phi-\phi_{X^{\prime}}\right)+2|X| \sin \left(\phi+\phi_{X}\right) \\
& =4 \cos ^{2} \phi \widehat{\delta M}-2 \sin \phi \cos \phi \widehat{\delta \Gamma}
\end{aligned}
$$

Note that $|\epsilon|$ drops out of the expression for $A_{C P T}$ as it should. In the long-time limit, we obtain

$$
A_{C P T}^{\mathrm{QM}} \rightarrow 4 \sin \phi \cos \phi \widehat{\delta M}+2 \cos ^{2} \phi \widehat{\delta \Gamma}
$$

Since the dynamical equations determining the density matrix do not manifestly possess the mimicking symmetry in Eq. (A28), one expects this mimicking phenomenon to break down in some observables. This is the case of $A_{C P T}$ where we find the asymptotic "mimic" result

$$
A_{C P T} \longrightarrow-4|\epsilon| \cos \phi \approx-6 \times 10^{-3},
$$


to be contrasted with the standard result of $A_{C P T}=0$. Experimentally, the CPLEAR Collaboration has measured this parameter to be $A_{C P T}^{\exp }=(-0.4 \pm 2.0 \pm 2.0 \pm 1.5) \times 10^{-3} \quad[3]$. Comparing the prediction in Eq. (A37) with the experimental data, we see that the "mimic" result appears disfavored by the $A_{C P T}$ measurement.

Finally, since $\Delta \rho_{12}^{(1)}=\Sigma \rho_{11}^{(1)}=\Sigma \rho_{22}^{(1)}=0$, the $A_{\Delta m}$ observable has the same first-order expression as in standard $C P$ violation: namely,

$$
A_{\Delta m}^{\mathrm{QM}}(t)=-\frac{2 e^{-\Gamma t} \cos \Delta m t}{e^{-\Gamma_{L} t}+e^{-\Gamma_{S} t}} .
$$

Since in this mechanism of $C P T$ violation, quantum mechanics is not violated, from the discussion in Sec. V B 2 we expect the parameter $\zeta$ to vanish. Indeed, using the above expressions for $\rho_{22}$, we find

$$
\begin{gathered}
c_{S}^{(0)}=\rho_{22}(0), \\
c_{L}^{(2)}=\rho_{11}(0)|X|^{2}, \\
c_{I}^{(1)}=\left|\rho_{12}(0)\right||X|,
\end{gathered}
$$

where we have also calculated the needed second-order (long-lived) terms in $\rho_{22}$. Moreover, the generic expression (120) gets modified in the interference term by the replacement: $\phi \rightarrow \phi+\phi_{X}+\phi_{12}$. It then immediately follows that $c_{I}^{2} /\left(c_{S} c_{L}\right)=\left|\rho_{12}(0)\right|^{2} /\left[\rho_{11}(0) \rho_{22}(0)\right]=1$, where we have made use of the $\operatorname{det} \rho(0)=0$ property. Therefore, as expected $\zeta=0$.

As in Sec. VI, we can derive indicative bounds on the $C P T$-violating parameters. The coefficient of the interference term in $A_{2 \pi}^{\mathrm{QM}}$ (A27) can be expressed as:

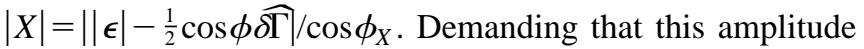
differ by less than $5 \%$ from the usual case, and with the $a$ priori knowledge that $\phi_{X}$ should be small (as we demonstrate below), we obtain $\frac{1}{2} \cos \phi|\widehat{\delta \Gamma}| / \epsilon \mid<0.05$, i.e.,

$$
|\widehat{\delta \Gamma}|<3 \times 10^{-4}, \quad|\delta \Gamma|<2 \times 10^{-18} \mathrm{GeV}
$$

We can obtain a bound on $\widehat{\delta M}$ by noticing the correspondence $\delta M \leftrightarrow-2 \beta$ that follows from Eqs. (45) and (A23) when the bound in Eq. (A42) holds. From Eq. (155), we then find

$$
\begin{gathered}
\widehat{\delta M}=(-4.0 \pm 4.4) \times 10^{-5}, \\
\delta M=(-3.0 \pm 3.2) \times 10^{-19} \mathrm{GeV} .
\end{gathered}
$$

Alternatively, the analogue of Eq. (157) is $\left|m_{K^{0}}-m_{K^{0}}\right| \approx|\delta M|$, which entails $|\delta M|<3.7 \times 10^{-19} \mathrm{GeV}$, once the $90 \%$ C.L. upper bound from E773 [23] is inserted.

\section{APPENDIX B: SECOND-ORDER CONTRIBUTIONS TO THE DENSITY MATRIX}

The second-order contributions to the density matrix in our quantum-mechanical-violating framework can be obtained by using Eq. (41) with the first-order inputs $\rho_{11,22,12}^{(1)}$ given in Eqs. (42)-(44). ${ }^{6}$ We obtain

$$
\rho_{11}^{(2)}=\sum_{k=1}^{7} c_{k}^{[11]} R_{k}^{[11]}(t),
$$

where the time-dependent $R_{k}^{[11]}(t)$ functions are given by

$$
\begin{gathered}
R_{1}^{[11]}(t)=e^{-\Gamma_{L} t}-e^{-\Gamma_{S} t}, \\
R_{2}^{[11]}(t)=t|\Delta \Gamma| e^{-\Gamma_{L} t}, \\
R_{3}^{[11]}(t)=-e^{-\Gamma t} \cos \left(\Delta m t-\delta \phi-\phi_{12}\right) \\
+e^{-\Gamma_{L^{t}} \cos \left(\delta \phi+\phi_{12}\right),} \\
R_{4}^{[11]}(t)=-e^{-\Gamma t} \sin (\Delta m t+\phi)+e^{-\Gamma_{L} t} \sin \phi, \\
R_{5}^{[11]}(t)=-e^{-\Gamma t}\left[\frac{|\Delta \Gamma| t}{2 \cos \phi} \cos \left(\Delta m t+\phi-\delta \phi-\phi_{12}\right)\right. \\
\left.+\cos \left(\Delta m t+2 \phi-\delta \phi-\phi_{12}\right)\right] \\
+e^{-\Gamma_{L} t} \cos \left(2 \phi-\delta \phi-\phi_{12}\right), \\
R_{6}^{[11]}(t)=-e^{-\Gamma t} \cos (\Delta m t+2 \phi-2 \delta \phi) \\
+e^{-\Gamma_{L} t} \cos (2 \phi-2 \delta \phi), \\
R_{7}^{[11]}(t)=-e^{-\Gamma t} \cos (\Delta m t-2 \delta \phi)+e^{-\Gamma_{L} t} \cos (2 \delta \phi),
\end{gathered}
$$

and the $c_{k}^{[11]}$ coefficients are

$$
\begin{gathered}
c_{1}^{[11]}=-\rho_{11}(0) \hat{\gamma}^{2}-\rho_{22}(0)|\epsilon|^{2} \frac{\cos (\phi+2 \delta \phi)}{\cos \phi \cos ^{2} \delta \phi} \\
-2\left|\rho_{12}(0)\right||\epsilon| \hat{\gamma} \frac{\cos \left(\phi+\delta \phi+\phi_{12}\right)}{\cos \delta \phi}, \\
c_{2}^{[11]}=\left[\hat{\gamma}^{2}-|\epsilon|^{2} \frac{\cos (\phi-2 \delta \phi)}{\cos \phi \cos ^{2} \delta \phi} \mid \rho_{11}(0),\right. \\
c_{3}^{[11]}=4|\epsilon| \hat{\gamma} \frac{\cos \phi}{\cos \delta \phi}\left|\rho_{12}(0)\right|, \\
c_{4}^{[11]}=-\frac{4 \hat{\alpha}|\epsilon|}{\tan \phi} \frac{\cos \left(\delta \phi-\phi \phi_{12}\right)}{\cos \delta \phi}\left|\rho_{12}(0)\right|, \\
c_{5}^{[11]}=4 \hat{\alpha}|\epsilon| \frac{\cos \phi}{\cos \delta \phi}\left|\rho_{12}(0)\right|, \\
c_{7}^{[11]}=\frac{2|\epsilon|^{2}}{\cos ^{2} \delta \phi} \rho_{22}(0) . \\
c^{[11]}=\frac{2|\epsilon|^{2}}{\cos ^{2} \delta \phi} \rho_{11}(0), \\
{ }^{[11} \delta
\end{gathered}
$$

\footnotetext{
${ }^{6}$ Expressions for $\rho_{22,12}^{(2)}$ valid for a particular choice of initial conditions were given in Ref. [16].
} 
Analogously,

$$
\rho_{22}^{(2)}=\sum_{k=1}^{7} c_{k}^{[22]} R_{k}^{[22]}(t)
$$

where the time-dependent $R_{k}^{[22]}(t)$ functions are given by

$$
\begin{gathered}
R_{1}^{[22]}(t)=e^{-\Gamma_{L} t}-e^{-\Gamma_{S} t} \\
R_{2}^{[22]}(t)=t|\Delta \Gamma| e^{-\Gamma_{S} t}
\end{gathered}
$$

$$
R_{3}^{[22]}(t)=e^{-\Gamma t} \cos \left(\Delta m t-\delta \phi-\phi_{12}\right)-e^{-\Gamma_{S} t} \cos \left(\delta \phi+\phi_{12}\right)
$$

$$
R_{4}^{[22]}(t)=e^{-\Gamma t} \sin (\Delta m t-\phi)+e^{-\Gamma_{S} t} \sin \phi
$$

$$
R_{5}^{[22]}(t)=e^{-\Gamma t}\left[\frac{|\Delta \Gamma| t}{2 \cos \phi} \cos \left(\Delta m t-\phi-\delta \phi-\phi_{12}\right)\right.
$$$$
\left.-\cos \left(\Delta m t-2 \phi-\delta \phi-\phi_{12}\right)\right]
$$$$
+e^{-\Gamma_{S} t} \cos \left(2 \phi+\delta \phi+\phi_{12}\right),
$$

$$
R_{6}^{[22]}(t)=-e^{-\Gamma t} \cos (\Delta m t-2 \delta \phi)+e^{-\Gamma_{S} t} \cos (2 \delta \phi),
$$

$$
\begin{aligned}
R_{7}^{[22]}(t)= & -e^{-\Gamma t} \cos (\Delta m t-2 \phi-2 \delta \phi) \\
& +e^{-\Gamma_{S} t} \cos (2 \phi+2 \delta \phi)
\end{aligned}
$$

and the $c_{k}^{[22]}$ coefficients are

$$
\begin{aligned}
& c_{1}^{[22]}=\rho_{22}(0) \hat{\gamma}^{2}+\rho_{11}(0)|\epsilon|^{2} \frac{\cos (\phi-2 \delta \phi)}{\cos \phi \cos ^{2} \delta \phi} \\
& -2\left|\rho_{12}(0)\right||\epsilon| \hat{\gamma} \frac{\cos \left(\phi-\delta \phi-\phi_{12}\right)}{\cos \delta \phi}, \\
& c_{2}^{[22]}=\left[-\hat{\gamma}^{2}+|\epsilon|^{2} \frac{\cos (\phi+2 \delta \phi)}{\cos \phi \cos ^{2} \delta \phi}\right] \rho_{22}(0), \\
& c_{3}^{[22]}=4|\epsilon| \hat{\gamma} \frac{\cos \phi}{\cos \delta \phi}\left|\rho_{12}(0)\right|, \\
& c_{4}^{[22]}=\frac{4 \hat{\alpha}|\epsilon|}{\tan \phi} \frac{\cos \left(\delta \phi-\phi_{12}\right)}{\cos \delta \phi}\left|\rho_{12}(0)\right|, \\
& c_{5}^{[22]}=-4 \hat{\alpha}|\epsilon| \frac{\cos \phi}{\cos \delta \phi}\left|\rho_{12}(0)\right|, \\
& c_{6}^{[22]}=\frac{2|\epsilon|^{2}}{\cos ^{2} \delta \phi} \rho_{11}(0), \\
& c_{7}^{[22]}=\frac{2|\epsilon|^{2}}{\cos ^{2} \delta \phi} \rho_{22}(0) .
\end{aligned}
$$

Finally,

$$
\begin{aligned}
\rho_{12}^{(2)}= & \frac{2 \hat{\alpha}}{\tan \phi}\left\{\frac{2 \hat{\alpha}}{\tan \phi}\left|\rho_{12}(0)\right| \sin \phi_{12} R_{1}^{[12]}(t, 0)-\frac{4 i \hat{\alpha}}{\tan \phi}\left|\rho_{12}(0)\right| R_{2}^{[12]}(t)\right. \\
& -\frac{|\epsilon|}{\cos \delta \phi}\left[\rho_{11}(0) R_{1}^{[12]}(t, \phi-\delta \phi)+\rho_{22}(0) R_{1}^{[12]}(t,-\phi-\delta \phi)\right] \\
& \left.+\frac{2 i|\epsilon| \sin \phi}{\cos \delta \phi}\left[\rho_{11}(0) \sin (\phi-\delta \phi) R_{3}^{[12]}(t)+\rho_{22}(0) \sin (\phi+\delta \phi) R_{4}^{[12]}(t)\right]\right\} \\
& +\frac{|\epsilon| e^{i \delta \phi}}{\cos \delta \phi}\left\{\hat{\gamma}\left[\rho_{22}(0)-\rho_{11}(0)\right]\left[R_{3}^{[12]}(t)+R_{4}^{[12]}(t)\right]+\frac{2|\epsilon|}{\cos \delta \phi}\left|\rho_{12}(0)\right|\left[i R_{1}^{[12]}\left(t,-\delta \phi-\phi_{12}\right)\right.\right. \\
& \left.\left.-\cos \left(\phi-\delta \phi-\phi_{12}\right) R_{3}^{[12]}(t)-\cos \left(\phi+\delta \phi+\phi_{12}\right) R_{4}^{[12]}(t)\right]\right\},
\end{aligned}
$$

where the time-dependent functions $R_{k}^{[12]}(t)$ are given by

$$
\begin{gathered}
R_{1}^{[12]}(t, a)=e^{-\Gamma t}\left[e^{i a} \sin \Delta m t-(\Delta m t) e^{-i \Delta m t-i a}\right], \\
R_{2}^{[12]}(t)=\frac{1}{4} e^{-\Gamma t}\left\{e^{-i \phi_{12}\left[\sin \Delta m t-(\Delta m t) e^{i \Delta m t}\right]+i(\Delta m t)^{2} e^{\left.-i \Delta m t+i \phi_{12}\right\}},}\right. \\
R_{3}^{[12]}(t)=e^{-i \phi}\left[e^{-\Gamma_{L} t}-e^{-(\Gamma+i \Delta m) t}\right], \\
R_{4}^{[12]}(t)=e^{i \phi}\left[e^{-\Gamma_{S} t}-e^{-(\Gamma+i \Delta m) t}\right] .
\end{gathered}
$$


[1] Particle Data Group, L. Montanet et al., Phys. Rev. D 50, 1173 (1994).

[2] G. Lüders, Ann. Phys. (N.Y.) 2, 1 (1957).

[3] CPLEAR Collaboration, R. Adler et al., in Proceedings of the XXVI International Conference on High Energy Physics, Dallas, Texas, 1992, edited by J. R. Sanford, AIP Conf. Proc. No. 272 (AIP, New York, 1993), p. 510; T. Ruf, "Measurements of $\mathrm{CP}$ and $\mathrm{T}$ violation parameters in the neutral kaon system at CPLEAR," report (unpublished); and (private communication).

[4] DAФNE Physics Handbook, edited by L. Maiani, L. Pancheri, and N. Paver (INFN, Frascati, 1992).

[5] N.W. Tanner and R.H. Dalitz, Ann. Phys. (N.Y.) 171, 463 (1986); C.D. Buchanan, R. Cousins, C. O. Dib, R.D. Peccei, and J. Quackenbush, Phys. Rev. D 45, 4088 (1992); C.O. Dib and R.D. Peccei, ibid. 46, 2265 (1992).

[6] J. Ellis, J.S. Hagelin, D.V. Nanopoulos, and M. Srednicki, Nucl. Phys. B241, 381 (1984).

[7] J. Ellis, N. E. Mavromatos, and D.V. Nanopoulos, Phys. Lett. B292, 37 (1992).

[8] S. W. Hawking, Commun. Math. Phys. 87, 395 (1982).

[9] J. Ellis, N.E. Mavromatos, and D.V. Nanopoulos, in From Supersymmetry to the Origin of Space-Time, Proceedings of the International School of Subnuclear Physics, Erice, Italy, 1993, Subnuclear Series Vol. 21 (World Scientific, Singapore, 1994); for a pedagogical review, see D. V. Nanopoulos, Riv. Nuovo Cimento 17, 1 (1994).

[10] D. J. Gross, Nucl. Phys. B236, 349 (1984).

[11] E. Witten, Commun. Math. Phys. 109, 525 (1987); H. Sonoda, Nucl. Phys. B326 , 135 (1989); A. Kostelecky and R. Potting, ibid. B359, 545 (1991).

[12] I. Antoniadis, C. Bachas, J. Ellis, and D.V. Nanopoulos, Phys. Lett. B 211, 393 (1988); Nucl. Phys. B328, 117 (1989); Phys. Lett. B 257, 278 (1991).

[13] F. David, Mod. Phys. Lett. A 3, 1651 (1988); J. Distler and H. Kawai, Nucl. Phys. B321, 509 (1989); N. E. Mavromatos and J. L. Miramontes, Mod. Phys. Lett. A 4, 1847 (1989); E. D'Hoker and P. S. Kurzepa, ibid. 5, 1411 (1990).

[14] R. Wald, Phys. Rev. D 21, 2742 (1980); D. N. Page, Gen.
Relativ. Gravit. 14, 299 (1982).

[15] J. Ellis, N. E. Mavromatos, and D.V. Nanopoulos, Phys. Lett. B 293, 142 (1992); Report No. CERN-TH.6755/92 (unpublished).

[16] J. L. Lopez in Recent Advances in the Superworld, Proceedings of the HARC Workshop, The Woodlands, 1993, edited by J. L. Lopez and D. V. Nanopoulos (World Scientific, Singapore, 1994), p. 272.

[17] P. Huet and M. Peskin, Nucl. Phys. B434, 3 (1995).

[18] V. A. Kostalecky and R. Potting, Phys. Rev. D 51, 3923 (1995).

[19] E. Shabalin, in Proceedings of the 30th Rencontre de Moriond, Méribel, France, 1994, edited by J. Tran Thanh Van (Editions Frontières, Gif-sur-Yvette, 1994).

[20] T. Nakada, in Lepton and Photon Interactions, Proceedings of the 16th International Symposium, Ithaca, New York, 1993, edited by P. S. Drell and D. L. Rubin, AIP Conf. Proc. No. 302 (AIP, New York, 1994), p. 425.

[21] See, e.g., E731 Collaboration, L. K. Gibbons et al., Phys. Rev. Lett. 70, 1199 (1993), and references therein.

[22] E773 Collaboration, G. Gollin and W. Hogan, in Proceedings of the 27th International Conference on High Energy Physics, Glasgow, Scotland, 1994, edited by P. J. Bussey and I. G. Knowles (IOP, London, 1995).

[23] E773 Collaboration, B. Schwingenheuer et al., Phys. Rev. Lett. 74, 4376 (1995).

[24] P. Eberhard, "Tests of Quantum Mechanics at a $\phi$ Factory," LBL-35983, contribution to the 2nd DAФNE Physics Handbook.

[25] W. C. Carithers et al., Phys. Rev. D 14, 290 (1976).

[26] R. A. Briere and L. H. Orr, Phys. Rev. D 40, 2269 (1989).

[27] CPLEAR Collaboration, J. Ellis, J. L. Lopez, N. E. Mavromatos, and D. V. Nanopoulos, Phys. Lett. B 364, 239 (1995).

[28] H. Lipkin, Phys. Lett. B 219, 474 (1988).

[29] J. Ellis, N. Mavromatos, and D. V. Nanopoulos, in Proceedings of the First International Conference on Phenomenology of Unification from Present to Future, Rome, 1994 (World Scientific, Singapore, 1995), p. 187. 